Article

\title{
Impact of DIY Home Manufacturing with 3D Printing on the Toy and Game Market
}

\author{
Emily E. Petersen ${ }^{1}$, Romain W. Kidd ${ }^{2}$ and Joshua M. Pearce ${ }^{1,3, *}$ \\ 1 Department of Material Science and Engineering, Michigan Technological University, Houghton, MI 49931, \\ USA; eepeters@mtu.edu \\ 2 MyMiniFactory, London E1 2JA, UK; romain@myminifactory.com \\ 3 Department of Electrical and Computer Engineering, Michigan Technological University, Houghton, \\ MI 49931, USA \\ * Correspondence: pearce@mtu.edu; Tel.: +1-906-487-1466
}

Received: 16 May 2017; Accepted: 6 July 2017; Published: 20 July 2017

\begin{abstract}
The 2020 toy and game market is projected to be US $\$ 135$ billion. To determine if 3D printing could affect these markets if consumers offset purchases by 3D printing free designs, this study investigates the 100 most popular downloaded designs at MyMiniFactory in a month. Savings are quantified for using a Lulzbot Mini 3D printer and three filament types: commercial filament, pellet-extruded filament, and post-consumer waste converted to filament with a recyclebot. Case studies probed the quality of: (1) six common complex toys; (2) Lego blocks; and (3) the customizability of open source board games. All filaments analyzed saved the user over $75 \%$ of the cost of commercially available true alternative toys and over $90 \%$ for recyclebot filament. Overall, these results indicate a single 3D printing repository among dozens is saving consumers well over $\$ 60$ million/year in offset purchases. The most common savings fell by $40 \%-90 \%$ in total savings, which came with the ability to make novel toys and games. The results of this study show consumers can generate higher value items for less money using the open source distributed manufacturing paradigm. It appears clear that consumer do-it-yourself (DIY) manufacturing is set to have a significant impact on the toy and game markets in the future.
\end{abstract}

Keywords: toy industry; additive manufacturing; 3D printing; consumer; economics; open-source

\section{Introduction}

After 20 years of legal intellectual monopoly, the fused filament fabrication (FFF) technology of additive manufacturing (AM), where a single layer of polymer is deposited after another, was unshackled by the open source release of the self-REPlicating RAPid prototyper 3D printer (RepRap) [1-3]. This open source hardware approach [4] led to a rapid technical evolution, which resulted in aggressive cost declines and the desktop 3D printer market emerged [5], dominated by various RepRap derivative machines [6,7]. Early adopters of these 3D printers were largely used for prototyping and the maker community, but this has morphed into peer production [8]. Digital peer-production with RepRaps found an eager audience among scientists to develop experimental tools [9-12]. In addition, teachers adopted the technology looking for high-quality educational experiences for their students [13-17] as well as those looking for economic sustainable development with appropriate technologies [18,19]. Sales of desktop 3D printers, however, are now moving towards the mass consumer market [6].

A pair of recent studies indicate that 3D printing technology is lucrative to adopt for average consumers. In the first study, the purchase of US $\$ 500$ components of a RepRap were justified by printing a handful of consumer products [20]. However, not all consumers are technically sophisticated makers able to build such a complex mechatronic technology alone, so a second study [21] looked 
at the use of an open source fully-assembled 3D printer (Lulzbot Mini, which retails for US\$1250). The costs of printing 26 free designs were compared with purchasing commercial equivalents and the study found that producing consumers would earn nearly a $1000 \%$ return on investment (ROI) from the purchase of a 3D printer over a printer lifetime of five years printing out only one product a week [21]. In addition, it appeared that consumers had already offset over $\$ 4$ million in purchases only from those random 26 products, which indicates that as 3D printer use in the home becomes more widespread, distributed manufacturing with open source designs could begin to have a significant macroeconomic impact [21]. There is significant skepticism of this potential [22] as the Foxconn CEO famously referred to 3D printers as "a gimmick" [23] and popular representations of 3D printers used only for toys in the home. However, the toy market is substantial and is not so easily dismissed, with the U.S. average spending per child on toys being \$371/year [24] resulting in a U.S. market of more than US $\$ 10$ billion/year [25] in 2013. In 2016, the NPD Group's Retail Tracking Service noted that the U.S. toy market had grown to $\$ 20.36$ billion [26] and the global toys and games market is projected to reach US $\$ 135$ billion by 2020 [27]. What are the effects on these markets if consumers are offsetting purchases of products like toys with 3D printing and free designs now?

To probe the potential economic impact of home use of 3D printing technology, this study closely investigates consumer use of a popular free website (MyMiniFactory) for 3D printable products. An economic analysis is performed from the perspective of users producing toys for themselves in their own homes. Specifically, the economic savings of the top 100 most popular designs as indicated by downloads (not views) on MyMiniFactory are quantified for January 2017. These savings are quantified using the sliced mass of filament and electricity consumption of a Lulzbot Mini 3D printer, the U.S. average electricity rate and three prices of filament: (1) the most popular filament sold on Amazon; (2) the use of a plastic extruder to make filament from commercial plastic pellets; and (3) the use of a home recyclebot to convert waste post-consumer waste into filament. The type of product is also evaluated and, because of the preponderance of products that can be classified as toys and games, three detailed case studies are undertaken. First, six common toys with equivalent products are evaluated in detail for functionality and value. Next, as Lego currently dominates the toy market [28] an economic evaluation is run using 3D printers only as Lego-compatible block factories. Finally, the costs and customizability are evaluated for an open source board game. Overall, the results are discussed in the context of distributed manufacturing in consumer homes and economics of do-it-yourself (DIY) production.

\section{Materials and Methods}

\subsection{Download Value Quantification}

A selection of items comprised of the 100 most downloaded files in January 2017 from MyMiniFactory, a repository for free 3D printable objects, was selected for analysis. The design and number of downloads $\left(\mathrm{N}_{\mathrm{d}}\right)$ is shown in Table 1 .

Table 1. Most popular designs downloaded on MyMiniFactory in January 2017.

\begin{tabular}{llc}
\hline \multicolumn{1}{c}{ Open Source 3D Printed Design } & \multicolumn{1}{c}{ URL (www.myminifactory.com/object) } & $\mathbf{N}_{\mathbf{d}}$ \\
\hline Pokemon Go aimer & pokeball-aimer-pokemon-go-23009 & 20583 \\
Clash of Clans barbarian & barbarian-lv-1-clash-of-clans-858 & 8107 \\
Voltron figure & voltron-defender-of-the-universe-22430 & 3881 \\
Overwatch tracer gun & tracer-gun-overwatch-19011 & 3602 \\
Overwatch reaper mask & reaper-mask-19004 & 3457 \\
Overwatch McCree revolver & updated-mccree-revolver-by-jeff-lagant-not-me-19543 & 3303 \\
Star Wars AT-AT & detailed-at-at-17606 & 3218 \\
Last Word Destiny Hand Cannon & destiny-last-word-exotic-hand-cannon-6546 & 3140 \\
Overwatch D.VA Light Gun & d-va-s-light-gun-18920 & 3135 \\
Overwatch Reaper Shotgun & reaper-s-hellfire-shotguns-overwatch-19096 & 2943 \\
Batman cowl & batman-cowl-20596 & 2926 \\
Destiny Hawkmoon gun & destiny-hawkmoon-exotic-hand-cannon-6545 & 2885 \\
\hline
\end{tabular}


Table 1. Cont.

\begin{tabular}{|c|c|c|}
\hline Open Source 3D Printed Design & URL (www.myminifactory.com/object) & $\mathbf{N}_{\mathbf{d}}$ \\
\hline Destiny thorn gun & thorn-from-destiny-4494 & 2846 \\
\hline Star Wars VII Storm Trooper Helmet & star-wars-storm-trooper-vii-fully-wearable-helmet-12992 & 2588 \\
\hline Kylo Ren helmet & jj-industries-kylo-ren-helmet-14106 & 2451 \\
\hline Wall outlet shelf & wall-outlet-shelf-6382 & 2350 \\
\hline Kylo Ren lightsaber & kylo-ren-s-lightsaber-star-wars-6791 & 2347 \\
\hline Blade Runner blaster & deckards-blaster-blade-runner-5694 & 2337 \\
\hline Fallout 3 T45-d helmet & fallout-3-t45-d-power-armour-helmet-15253 & 2318 \\
\hline Venus de Milo figurine & venus-de-milo-at-louvre-paris-1657 & 2073 \\
\hline Warcraft Frostmourne sword & frostmourne-from-warcraft- 4156 & 1999 \\
\hline 3DR Iris+ quadcopter & 3drobotics-iris-19615 & 1871 \\
\hline Pieta figurine & pieta-in-st-peter-s-basilica-vatican-3796 & 1862 \\
\hline P08 Luger gun & p08-luger-functional-assembly- 5545 & 1798 \\
\hline Game of Thrones iron throne & game-of-thrones-iron-throne-1945 & 1786 \\
\hline The Thinker figurine & the-thinker-at-the-muse-rodin-france-2127 & 1755 \\
\hline Overwatch McCree Peacemaker gun & mccree-peacemaker-overwatch-19152 & 1723 \\
\hline Fallout 4 Pipboy 3000 MkIV & fallout-4-pipboy-3000-mkiv-16884 & 1705 \\
\hline Articulated lamp & articulated-lamp-6790 & 1704 \\
\hline Overwatch throwing star & genji-s-shuriken-18918 & 1695 \\
\hline Strong bolt & support-free-bolt-1281 & 1571 \\
\hline Mazigner Z Super Robot & mazigner-z-super-robot-24533 & 1570 \\
\hline Secret shelf & secret-shelf-3504 & 1564 \\
\hline Sombra pistol & sombra-s-machine-pistol-25186 & 1544 \\
\hline Han Solo blaster & han-solo-s-blaster-star-wars-1546 & 1520 \\
\hline Overwatch D.VA headset & d-va-headset-22077 & 1502 \\
\hline Overwatch Mercy staff & mercy-s-staff-22079 & 1484 \\
\hline Overwatch Mercy blaster & mercy-s-caduceus-blaster-18912 & 1481 \\
\hline Han Solo blaster & hans-solo-blaster-2488 & 1461 \\
\hline Gears of War Chainsaw gun & gears-of-war-lancer-chainsaw-gun-11478 & 1437 \\
\hline Destiny Duke MK.44 gun & duke-mk-44-hand-cannon-from-destiny-2140 & 1418 \\
\hline Fallout 4 Laser pistol & fallout-4-laser-pistol-18978 & 1417 \\
\hline Overwatch loot box & overwatch-loot-box-21670 & 1412 \\
\hline Cat at British Museum & gayer-anderson-cat-at-the-british-museum-london-4010 & 1364 \\
\hline Halo 5 assault rifle & halo-5-assault-rifle-11734 & 1334 \\
\hline Portal gun & portal-gun-18342 & 1328 \\
\hline Destiny hawkmoon gun & hawkmoon-from-destiny-full-scale-and-moving- 6863 & 1327 \\
\hline Clash of Clans figurine & p-e-k-k-a-lv-1-clash-of-clans-857 & 1314 \\
\hline Harry Potter elder wand & dumbledore-s-elder-wand-2057 & 1299 \\
\hline Groot flower pot & baby-groot-flower-pot-gardens-of-the-galaxy-2-26442 & 1269 \\
\hline Melted Darth Vader mask & darth-vader-melted-mask- 6685 & 1264 \\
\hline Fallout $410 \mathrm{~mm}$ pistol & fallout-4-10mm-pistol-10475 & 1264 \\
\hline Starcraft Kerrigan statue & starcraft-kerrigan-statue-10432 & 1263 \\
\hline Destiny ghost & destiny-ghost- 6038 & 1248 \\
\hline Lich king figurine & the-lich-king-6174 & 1212 \\
\hline Micro game bit & micro-bit-game-bit-13822 & 1199 \\
\hline Ant Man helmet & ant-man-mask-wearable-5322 & 1193 \\
\hline Game of Thrones House emblem & house-stark-game-of-thrones-1154 & 1182 \\
\hline Planetary gears & planetary-gears-1557 & 1163 \\
\hline Clash of Clans king figure & barbarian-king-clash-of-clans-871 & 1160 \\
\hline Tooth toothbrush holder & the-big-tooth-2-0-5759 & 1141 \\
\hline Halo 3 ODST helmet & halo-3-odst-helmet-wearable-cosplay-17614 & 1134 \\
\hline Fallout 4 protectron figure & fallout-4-protectron-action-figure-15585 & 1129 \\
\hline BFG Doom & bfg-21395 & 1122 \\
\hline Discobolus figurine & discobolus-at-the-british-museum-london-7896 & 1118 \\
\hline Anonymous mask & guy-fawkes-anonymous-mask-2582 & 1092 \\
\hline Guardian of the Galaxy Star Lord mask & guardians-of-the-galaxy-star-lord-s-mask-version-2-3045 & 1091 \\
\hline Pokeball & pokeball-high-detail-version- 23506 & 1064 \\
\hline Witcher 3 wall plaque & the-witcher-3-wall-plaque- 8882 & 1063 \\
\hline Overwatch widowmaker rifle & overwatch-widowmaker-sniper-rifle-21702 & 1029 \\
\hline Fallout 4 combat rifle and shotgun & fallout-4-combat-rifle-and-combat-shotgun- 18428 & 1025 \\
\hline Michelangelo's David & michelangelo-s-david-in-florence-italy-2052 & 1023 \\
\hline Destiny ghost & ghost-destiny-2396 & 1022 \\
\hline Triceratops skull & triceratops-skull-in-colorado-usa- 6225 & 1019 \\
\hline Skull ring & skull-ring-20782 & 1014 \\
\hline Star Wars NN-44 Rey's Blaster & star-wars-nl-44-reys-blaster-17422 & 1012 \\
\hline Joker mask & joker-mask-9743 & 1010 \\
\hline Fork/spoon support for disability & fork-and-spoon-support-for-person-with-disabilities- 5480 & 1007 \\
\hline BFG Doom & bfg-parts-19092 & 1006 \\
\hline Gryffindor coat of arms & gryffindor-coat-of-arms-wall-desk-display-harry-potter-11834 & 994 \\
\hline Destiny Gjallahorn & gjallarhorn-2-0-destiny-19160 & 985 \\
\hline Pokemon figurines & low-poly-pokemon-collection- 15905 & 983 \\
\hline Rubik's cube & 3d-printable-rubik-s-cube- 9734 & 980 \\
\hline Destiny bad juju pulse rifle & destiny-s-bad-juju-exotic-pulse-rifle- 6618 & 977 \\
\hline Fallout 4 Kellogg's pistol & kellogg-s-pistol-fallout-4-21556 & 975 \\
\hline Dobby the Elf figurine & dobby-from-harry-potter-full-model-3294 & 970 \\
\hline Statue of Liberty figurine & statue-of-liberty-in-manhatten-new-york-2077 & 960 \\
\hline Nutcracker & nutcracker-v2-4361 & 946 \\
\hline Minions figurines & minion-movie-trio- 10140 & 940 \\
\hline Destiny sleeper simulant & the-sleeper-simulant-from-destiny-14769 & 930 \\
\hline
\end{tabular}


Table 1. Cont.

\begin{tabular}{lll}
\hline \multicolumn{1}{c}{ Open Source 3D Printed Design } & \multicolumn{1}{c}{ URL (www.myminifactory.com/object) } & $\mathbf{N}_{\mathbf{d}}$ \\
\hline Frozen Elsa figurine & elsa-from-disney-s-frozen-6573 & 919 \\
Star Wars storm trooper rifle & blaster-rifle-star-wars-storm-trooper-12097 & 914 \\
Vitruvian Man scuplture & the-vetruvian-man-sculpture-at-belgrave-square-london-1669 & 908 \\
Robocop ED209 figure & ed209-from-robocop-5090 & 904 \\
Harry Potter wand & harry-potter-s-wand-10391 & 898 \\
Cable guards & icableguards-21235 & 894 \\
Game of Thrones dice cup & stark-dice-cup-1847 & 893 \\
Overwatch McCree flashbang & mccree-flashbang-from-overwatch-21595 & 890 \\
B2 bomber glider & b2-stealth-bomber-glider-improved-flight-powered-by-an-elastic-band-13337 & 886 \\
Star Wars X Wing helmet & x-wing-pilot-helmet-starwars-episode-vii-the-force-awakens-9074 & 859 \\
\hline
\end{tabular}

The items were uploaded into Cura 15.04.6 (Ultimaker, Geldermalsen, The Netherlands) [29] and the resulting data regarding estimated print time, item weight, and length of the filament used were recorded. In addition, a 3-mm poly lactic acid (PLA) was selected as the filament because it is the most common household consumer 3D printing material and is available from most 3D printing suppliers. PLA has gained prominence, as not only does it demonstrate less warping during printing than other materials such as the second most common 3D printing plastic (ABS, acrylonitrile butadiene styrene), but the emissions during printing are less pungent [30,31]. Furthermore, PLA is made from corn-based resin, making it non-toxic, biodegradable, and able to be produced in environmentally friendly, renewable processes [32]. The items were then categorized into three groups using commercial PLA: (1) those that saved the consumer money when compared to a commercially available alternative product; (2) those that lost money because a less expensive product was available; and (3) those for which there was no alternative product. Items ranged from action figures and masks to non-toy items and cosplay paraphernalia as seen in Table 1.

A commercial price for each product was found primarily on Walmart.com and supplemented using Google Shopping. Associated shipping costs were excluded from the analysis for both purchasing and distributed manufacturing (e.g., no shipping charges included for plastic filament). Following [21], the operating cost (O) for the Lulzbot Mini [33] was calculated using electricity and filament consumption during printing with $15 \%$ infill. The average electric rate in the United States was found to be $\$ 0.1267$ per $\mathrm{kWh}$ for the residential sector [34]. A sensitivity was run on the cost of the filament $\left(\mathrm{C}_{\mathrm{F}(\text { source })}\right)$ using:

(1) the most popular filament sold on Amazon.com, $\mathrm{C}_{\mathrm{F}(\text { filament) }}$ is US $\$ 23 / \mathrm{kg}$ [35];

(2) the use of a plastic extruder such as a commercial systems (e.g., Filastruder, FilaFab, Noztek, Filabot, EWE, Extrusionbot, Filamaker, Strooder, Felfil, ExtrusionBot) to make filament from commercial PLA pellets (source) $\mathrm{C}_{\mathrm{F} \text { (pellets) }}$ is US $\$ 5.50 / \mathrm{kg}$ [36];

(3) the use of a home recyclebot (waste plastic extruder) to convert waste post-consumer waste

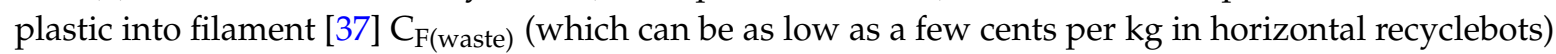
for ABS in vertical recyclebot in another study it was found to be US\$2.16/ $\mathrm{kg}$ [38] and will be used here to be conservative.

The operating cost, $\mathrm{O}$, of a 3D printer was calculated as follows:

$$
\mathrm{O}=\mathrm{EC}_{\mathrm{E}}+\frac{\mathrm{C}_{\mathrm{F}} \mathrm{m}_{\mathrm{f}}}{1000}[\mathrm{USD}]
$$

where $\mathrm{E}$ is the energy consumed in $\mathrm{kWh}, \mathrm{C}_{\mathrm{E}}$ is the average rate of electricity in the United States in $\mathrm{USD} / \mathrm{kWh}, \mathrm{C}_{\mathrm{F}}$ is the cost of a given filament in $\mathrm{USD} / \mathrm{kg}$, and $\mathrm{m}_{\mathrm{f}}$ is the mass of the filament in grams consumed during printing. The marginal savings on each project, $\mathrm{Cs}$, is given by:

$$
\mathrm{C}_{\mathrm{S}}=\mathrm{C}_{\mathrm{C}}-\mathrm{O} \quad[\mathrm{USD}],
$$


where $C_{C}$ is the cost of the commercially available product and the marginal percent change, $\mathrm{P}$, between the cost to print a product and the commercially available product was calculated as follows:

$$
\mathrm{P}=\frac{\mathrm{C}_{\mathrm{S}}}{\mathrm{C}_{\mathrm{C}}} \times 100[\%]
$$

where $C_{C}$ is the cost for the commercial product at either the high or low price. Finally, the value obtained from a free and open source 3D printable design can be determined from the $[39,40]$ at a specific time $(\mathrm{t})$ :

$$
\mathrm{V}_{\mathrm{D}}(\mathrm{t})=\mathrm{C}_{\mathrm{S}} \times \mathrm{P} \times \mathrm{N}_{\mathrm{d}}(\mathrm{t})[\mathrm{USD}],
$$

This value is determined by the number of downloads $\left(\mathrm{N}_{\mathrm{d}}\right)$ during January 2017 and $\mathrm{P}$ where is the percent of downloads resulting in a print. It should be pointed out that $\mathrm{P}$ is subject to error as downloading a design does not guarantee manufacturing. On the other (more likely) hand, a single download could be fabricated many times, traded via email, memory stick or posted on P2P websites that are beyond conventional tracking. Here, to remain conservative, $\mathrm{P}$ is assumed to be 1 and the total savings found over MyMiniFactory in 1 month on the top 100 downloaded items is determined by:

$$
\mathrm{V}_{\mathrm{DT}}(\mathrm{t})=\sum_{i=0}^{100} \mathrm{~V}_{\mathrm{D}}(\mathrm{t})[\mathrm{USD}]
$$

Three case studies are then presented to probe the economics of individual types of toys and games.

\subsection{Case Studies}

\subsubsection{Six Toy Product Comparisons}

As is clear from Table 1, the most popular types of 3D printable design are niche-community toys (e.g., gamers, cosplay, etc.). Six more common toy products with existing free designs on MyMiniFactory were selected for more detailed comparisons attractive to a wider audience. Printing costs for these toys were estimated using $\$ 24 / \mathrm{kg}$ filament and the closest commercial equivalent was found online. The toys are compared visually and the savings percent is calculated by Equation (3), where it is conservatively assumed to be zero (e.g., the electricity cost was ignored).

\subsubsection{Lego Case Study}

Lego is one of the top five leading toy industry manufacturers with $5 \%$ of the market [41]. Lego's signature toy is manufactured to exacting specifications from ABS, a common consumer polymer. In a past study, on average, a single Lego piece costs $\$ 0.104$ USD, and the average cost of a Lego set without pieces, thereby the cost of the box and printed instructions, is $\$ 7.34$ [42]. It should be pointed out that, in consideration of their larger size intended for small children, Duplo blocks and thus Duplo sets are more expensive at $\$ 0.63$ per brick. When grouped into themes, it was found that Lego city-themed and architecture-themed sets had the lowest base cost while Marvel-themed sets had a base cost of $\$ 3.61$ per piece [42].

The study by Allain is expanded here to look at the cost per block in the 10 most popular Lego kits at Wal-Mart. The cost to print a $3 \times 2$ block is then determined. The 3D printed blocks are then tested and compared to Lego blocks and generic Lego-compatible commercial blocks on a quality and price basis.

\subsubsection{Board Game Comparison}

The hobby games market is more than US\$1.2 billion, with the hobby game board market having grown 56\% from $\$ 160$ million in 2014 to $\$ 250$ million in 2015 [43]. ICv2 CEO Milton Griepp has claimed that there is often a transition from digital games to tabletop games when users are more interested in 
face-to-face interaction [44]. The global board games market is expected to experience a $29 \%$ growth over the period from 2017 to 2021, largely due to increased popularity amongst the adult consumer demographic [45]. Three market trends-the growth of organized retail, increase in projects funded through crowdsourcing, and game evolution with time- - have been cited as driving forces behind this projected growth. Here again, 3D printing can be used to manufacture board games at home. Technavio points out that 3D printing have been shown to boost the sales of board games [45]. To demonstrate the feasibility and compare the costs to a traditional board game, an open source game, 'Save the planet' board game hosted on Appropedia [46] has been selected. The costs to commercially print out the board and the cards is quantified from a professional print shop and compared to 2D printing on paper by a home printer. Then, the costs for the dice and four player pieces are determined by printing and weighing on a digital scale $( \pm 0.1 \mathrm{~g})$ and assuming filament costs from above. These costs are compared to the top ten board games by sales on Amazon [47].

\section{Results and Discussion}

\subsection{Downloaded Value}

\subsubsection{Microeconomic Advantages of Home Manufacturing}

When the cost was calculated for each designed item printed with each of the filaments as described in the Methods section, the recyclebot-produced filament proved to demonstrate the greatest savings for the user. By using an at-home recyclebot or extruder-produced pellets, printing costs can be significantly reduced, and even using more expensive filament found on Amazon demonstrates savings when compared to equivalent, commercially available products. Table 2 shows the cost of each design printed in each of the three filaments as well as the cost of the commercially available product equivalent. On average, printing the items in Amazon filament cost $\$ 9.28$ while printing in pellets and recyclebot filament cost on average $\$ 2.59$ and $\$ 1.31$, respectively. Table 2 shows a clear financial advantage to the consumer in printing items as opposed to purchasing them as shown by the printing cost with recyclebot filament costing on average a mere $3.09 \%$ of the cost to purchase. Even when compared to the more expensive Amazon filament, printing costs, on average, only $22 \%$ of the cost to purchase. It should also be noted here (and is shown in Section 3.3) that for applications such as toys there is not a noticeable tradeoff between quality and cost of filament. A tuned recyclebot can produce filament with equivalent visual quality to commercial filament.

Table 2. Cost of producing toys and benchmark purchase price (US \$).

\begin{tabular}{|c|c|c|c|c|c|}
\hline Design & Mass (g) & $\begin{array}{l}\text { Cost: } \\
\text { Commercial } \\
\text { Filament }\end{array}$ & Cost: Pellet & $\begin{array}{c}\text { Cost: } \\
\text { Recyclebot }\end{array}$ & $\begin{array}{c}\text { Commercially } \\
\text { Available } \\
\text { Alternative } \\
\text { (USD) }\end{array}$ \\
\hline Pokemon Go aimer & 27 & $\$ 0.66$ & $\$ 0.18$ & $\$ 0.09$ & $\$ 4.99$ \\
\hline Clash of Clans barbarian & 21 & $\$ 0.51$ & $\$ 0.14$ & $\$ 0.07$ & $\$ 19.00$ \\
\hline Voltron figure & 1247 & $\$ 30.26$ & $\$ 8.44$ & $\$ 4.27$ & $\$ 9.99$ \\
\hline Overwatch tracer gun & 190 & $\$ 4.61$ & $\$ 1.29$ & $\$ 0.65$ & $\$ 26.59$ \\
\hline Overwatch reaper mask & 333 & $\$ 8.08$ & $\$ 2.25$ & $\$ 1.14$ & $\$ 25.00$ \\
\hline Star Wars AT-AT & 683 & $\$ 16.57$ & $\$ 4.62$ & $\$ 2.34$ & $\$ 7.50$ \\
\hline Last Word Destiny Hand Cannon & 367 & $\$ 8.91$ & $\$ 2.48$ & $\$ 1.26$ & $\$ 100.00$ \\
\hline Overwatch D.VA Light Gun & 261 & $\$ 6.33$ & $\$ 1.77$ & $\$ 0.89$ & $\$ 55.00$ \\
\hline Overwatch Reaper Shotgun & 269 & $\$ 6.53$ & $\$ 1.82$ & $\$ 0.92$ & $\$ 40.38$ \\
\hline Batman cowl & 147 & $\$ 3.57$ & $\$ 0.99$ & $\$ 0.50$ & $\$ 6.76$ \\
\hline Destiny Hawkmoon gun & 473 & $\$ 11.48$ & $\$ 3.20$ & $\$ 1.62$ & $\$ 99.99$ \\
\hline Destiny thorn gun & 425 & $\$ 10.31$ & $\$ 2.88$ & $\$ 1.46$ & $\$ 17.99$ \\
\hline Star Wars VII Storm Trooper Helmet & 948 & $\$ 23.01$ & $\$ 6.42$ & $\$ 3.25$ & $\$ 20.20$ \\
\hline Kylo Ren helmet & 550 & $\$ 13.35$ & $\$ 3.72$ & $\$ 1.88$ & $\$ 4.08$ \\
\hline Wall outlet shelf & 69 & $\$ 1.67$ & $\$ 0.47$ & $\$ 0.24$ & $\$ 10.00$ \\
\hline Kylo Ren lightsaber & 319 & $\$ 7.74$ & $\$ 2.16$ & $\$ 1.09$ & $\$ 20.00$ \\
\hline Blade Runner blaster & 249 & $\$ 6.04$ & $\$ 1.68$ & $\$ 0.85$ & $\$ 12.99$ \\
\hline Fallout 3 T45-d helmet & 1586 & $\$ 38.49$ & $\$ 10.73$ & $\$ 5.44$ & $\$ 185.91$ \\
\hline Venus de Milo figurine & 28 & $\$ 0.68$ & $\$ 0.19$ & $\$ 0.10$ & $\$ 9.00$ \\
\hline
\end{tabular}


Table 2. Cont.

\begin{tabular}{|c|c|c|c|c|c|}
\hline Design & Mass (g) & $\begin{array}{c}\text { Cost: } \\
\text { Commercial } \\
\text { Filament }\end{array}$ & Cost: Pellet & $\begin{array}{c}\text { Cost: } \\
\text { Recyclebot }\end{array}$ & $\begin{array}{c}\text { Commercially } \\
\text { Available } \\
\text { Alternative } \\
\text { (USD) }\end{array}$ \\
\hline Warcraft Frostmourne sword & 1233 & $\$ 29.92$ & $\$ 8.34$ & $\$ 4.23$ & $\$ 133.00$ \\
\hline 3DR Iris+ quadcopter & 255 & $\$ 6.19$ & $\$ 1.73$ & $\$ 0.87$ & $\$ 13.89$ \\
\hline Pieta figurine & 164 & $\$ 3.98$ & $\$ 1.11$ & $\$ 0.56$ & $\$ 16.88$ \\
\hline P08 Luger gun & 138 & $\$ 3.35$ & $\$ 0.93$ & $\$ 0.47$ & $\$ 79.00$ \\
\hline Game of Thrones iron throne & 124 & $\$ 3.01$ & $\$ 0.84$ & $\$ 0.42$ & $\$ 15.25$ \\
\hline The Thinker figurine & 92 & $\$ 2.23$ & $\$ 0.62$ & $\$ 0.32$ & $\$ 22.32$ \\
\hline Overwatch McCree Peacemaker gun & 301 & $\$ 7.30$ & $\$ 2.04$ & $\$ 1.03$ & $\$ 69.99$ \\
\hline Fallout 4 Pipboy 3000 MkIV & 884 & $\$ 21.45$ & $\$ 5.98$ & $\$ 3.03$ & $\$ 74.99$ \\
\hline Articulated lamp & 180 & $\$ 4.37$ & $\$ 1.22$ & $\$ 0.62$ & $\$ 7.97$ \\
\hline Overwatch throwing star & 13 & $\$ 0.32$ & $\$ 0.09$ & $\$ 0.04$ & $\$ 6.99$ \\
\hline Strong bolt & 9 & $\$ 0.22$ & $\$ 0.06$ & $\$ 0.03$ & $\$ 19.14$ \\
\hline Mazigner Z Super Robot & 117 & $\$ 2.84$ & $\$ 0.79$ & $\$ 0.40$ & $\$ 9.99$ \\
\hline Sombra pistol & 495 & $\$ 12.01$ & $\$ 3.35$ & $\$ 1.70$ & $\$ 85.00$ \\
\hline Han Solo blaster & 216 & $\$ 5.24$ & $\$ 1.46$ & $\$ 0.74$ & $\$ 10.12$ \\
\hline Overwatch D.VA headset & 65 & $\$ 1.58$ & $\$ 0.44$ & $\$ 0.22$ & $\$ 18.99$ \\
\hline Overwatch Mercy staff & 1768 & $\$ 42.90$ & $\$ 11.96$ & $\$ 6.06$ & $\$ 165.89$ \\
\hline Overwatch Mercy blaster & 411 & $\$ 9.97$ & $\$ 2.78$ & $\$ 1.41$ & $\$ 19.49$ \\
\hline Gears of War Chainsaw gun & 1556 & $\$ 37.76$ & $\$ 10.53$ & $\$ 5.33$ & $\$ 199.99$ \\
\hline Destiny Duke MK.44 gun & 325 & $\$ 7.89$ & $\$ 2.20$ & $\$ 1.11$ & $\$ 125.00$ \\
\hline Fallout 4 Laser pistol & 1322 & $\$ 32.08$ & $\$ 8.95$ & $\$ 4.53$ & $\$ 89.99$ \\
\hline Overwatch loot box & 124 & $\$ 3.01$ & $\$ 0.84$ & $\$ 0.42$ & $\$ 8.98$ \\
\hline Cat at British Museum & 62 & $\$ 1.50$ & $\$ 0.42$ & $\$ 0.21$ & $\$ 75.99$ \\
\hline Halo 5 assault rifle & 2295 & $\$ 55.69$ & $\$ 15.53$ & $\$ 7.86$ & $\$ 50.00$ \\
\hline Portal gun & 1528 & $\$ 37.08$ & $\$ 10.34$ & $\$ 5.24$ & $\$ 279.99$ \\
\hline Clash of Clans figurine & 2 & $\$ 0.05$ & $\$ 0.01$ & $\$ 0.01$ & $\$ 29.99$ \\
\hline Harry Potter elder wand & 17 & $\$ 0.41$ & $\$ 0.12$ & $\$ 0.06$ & $\$ 4.79$ \\
\hline Groot flower pot & 57 & $\$ 1.38$ & $\$ 0.39$ & $\$ 0.20$ & $\$ 8.99$ \\
\hline Melted Darth Vader mask & 172 & $\$ 4.17$ & $\$ 1.16$ & $\$ 0.59$ & $\$ 8.00$ \\
\hline Fallout 4 10mm pistol & 793 & $\$ 19.24$ & $\$ 5.37$ & $\$ 2.72$ & $\$ 24.99$ \\
\hline Starcraft Kerrigan statue & 112 & $\$ 2.72$ & $\$ 0.76$ & $\$ 0.38$ & $\$ 55.79$ \\
\hline Destiny ghost & 116 & $\$ 2.81$ & $\$ 0.78$ & $\$ 0.40$ & $\$ 7.00$ \\
\hline Lich king figurine & 487 & $\$ 11.82$ & $\$ 3.30$ & $\$ 1.67$ & $\$ 15.00$ \\
\hline Ant Man helmet & 367 & $\$ 8.91$ & $\$ 2.48$ & $\$ 1.26$ & $\$ 44.59$ \\
\hline Game of Thrones House emblem & 34 & $\$ 0.83$ & $\$ 0.23$ & $\$ 0.12$ & $\$ 9.95$ \\
\hline Clash of Clans king figure & 24 & $\$ 0.58$ & $\$ 0.16$ & $\$ 0.08$ & $\$ 19.00$ \\
\hline Tooth toothbrush holder & 120 & $\$ 2.91$ & $\$ 0.81$ & $\$ 0.41$ & $\$ 2.00$ \\
\hline Halo 3 ODST helmet & 1118 & $\$ 27.13$ & $\$ 7.57$ & $\$ 3.83$ & $\$ 9.99$ \\
\hline Fallout 4 protectron figure & 98 & $\$ 2.38$ & $\$ 0.66$ & $\$ 0.34$ & $\$ 24.99$ \\
\hline Discobolus figurine & 35 & $\$ 0.85$ & $\$ 0.24$ & $\$ 0.12$ & $\$ 7.25$ \\
\hline Anonymous mask & 383 & $\$ 9.29$ & $\$ 2.59$ & $\$ 1.31$ & $\$ 4.95$ \\
\hline Guardian of the Galaxy Star Lord mask & 351 & $\$ 8.52$ & $\$ 2.38$ & $\$ 1.20$ & $\$ 5.99$ \\
\hline Pokeball & 221 & $\$ 5.36$ & $\$ 1.50$ & $\$ 0.76$ & $\$ 1.00$ \\
\hline Witcher 3 wall plaque & 100 & $\$ 2.43$ & $\$ 0.68$ & $\$ 0.34$ & $\$ 45.00$ \\
\hline Overwatch widowmaker rifle & 1364 & $\$ 33.10$ & $\$ 9.23$ & $\$ 4.67$ & $\$ 145.89$ \\
\hline Fallout 4 combat rifle and shotgun & 351 & $\$ 8.52$ & $\$ 2.38$ & $\$ 1.20$ & $\$ 85.00$ \\
\hline Michelangelo's David & 75 & $\$ 1.82$ & $\$ 0.51$ & $\$ 0.26$ & $\$ 29.93$ \\
\hline Triceratops skull & 66 & $\$ 1.60$ & $\$ 0.45$ & $\$ 0.23$ & $\$ 40.00$ \\
\hline Skull ring & 5 & $\$ 0.12$ & $\$ 0.03$ & $\$ 0.02$ & $\$ 9.95$ \\
\hline Star Wars NN-44 Rey's Blaster & 141 & $\$ 3.42$ & $\$ 0.95$ & $\$ 0.48$ & $\$ 45.00$ \\
\hline Joker mask & 751 & $\$ 18.22$ & $\$ 5.08$ & $\$ 2.57$ & $\$ 11.56$ \\
\hline Fork/spoon support for disability & 17 & $\$ 0.41$ & $\$ 0.12$ & $\$ 0.06$ & $\$ 11.00$ \\
\hline Gryffindor coat of arms & 48 & $\$ 1.16$ & $\$ 0.32$ & $\$ 0.16$ & $\$ 8.00$ \\
\hline Pokemon figurines & 63 & $\$ 1.53$ & $\$ 0.43$ & $\$ 0.22$ & $\$ 30.00$ \\
\hline Rubik's cube & 12 & $\$ 0.29$ & $\$ 0.08$ & $\$ 0.04$ & $\$ 4.99$ \\
\hline Fallout 4 Kellogg's pistol & 152 & $\$ 3.69$ & $\$ 1.03$ & $\$ 0.52$ & $\$ 100.00$ \\
\hline Dobby the Elf figurine & 365 & $\$ 8.86$ & $\$ 2.47$ & $\$ 1.25$ & $\$ 31.36$ \\
\hline Statue of Liberty figurine & 85 & $\$ 2.06$ & $\$ 0.58$ & $\$ 0.29$ & $\$ 6.44$ \\
\hline Nutcracker & 61 & $\$ 1.48$ & $\$ 0.41$ & $\$ 0.21$ & $\$ 4.99$ \\
\hline Minions figurines & 121 & $\$ 2.94$ & $\$ 0.82$ & $\$ 0.41$ & $\$ 26.80$ \\
\hline Destiny sleeper simulant & 1274 & $\$ 30.92$ & $\$ 8.62$ & $\$ 4.37$ & $\$ 211.66$ \\
\hline Frozen Elsa figurine & 33 & $\$ 0.80$ & $\$ 0.22$ & $\$ 0.11$ & $\$ 7.99$ \\
\hline Star Wars storm trooper rifle & 486 & $\$ 11.79$ & $\$ 3.29$ & $\$ 1.67$ & $\$ 170.00$ \\
\hline Vitruvian Man scuplture & 12 & $\$ 0.29$ & $\$ 0.08$ & $\$ 0.04$ & $\$ 31.49$ \\
\hline Robocop ED209 figure & 736 & $\$ 17.86$ & $\$ 4.98$ & $\$ 2.52$ & $\$ 21.99$ \\
\hline Harry Potter wand & 8 & $\$ 0.19$ & $\$ 0.05$ & $\$ 0.03$ & $\$ 13.55$ \\
\hline Cable guards & 7 & $\$ 0.17$ & $\$ 0.05$ & $\$ 0.02$ & $\$ 1.25$ \\
\hline Game of Thrones dice cup & 59 & $\$ 1.43$ & $\$ 0.40$ & $\$ 0.20$ & $\$ 8.32$ \\
\hline B2 bomber glider & 20 & $\$ 0.49$ & $\$ 0.14$ & $\$ 0.07$ & $\$ 10.57$ \\
\hline Star Wars X Wing helmet & 949 & $\$ 23.03$ & $\$ 6.42$ & $\$ 3.25$ & $\$ 41.40$ \\
\hline Hawkmoon \#2 & 621 & $\$ 15.07$ & $\$ 4.20$ & $\$ 2.13$ & $\$ 99.99$ \\
\hline Destiny ghost \#2 & 48 & $\$ 1.16$ & $\$ 0.32$ & $\$ 0.16$ & $\$ 7.00$ \\
\hline Average & 382.29 & $\$ 9.28$ & $\$ 2.59$ & $\$ 1.31$ & $\$ 42.44$ \\
\hline Total & 34,406 & $\$ 834.93$ & $\$ 232.83$ & $\$ 117.91$ & $\$ 3819.59$ \\
\hline
\end{tabular}


Table 3 shows the cost and percent savings calculated for each design in each of the three filament types when compared to their respective commercially available alternative products. As expected based on the results in Table 2, printed items using recyclebot-made filament saved the user significantly more money than printed items using commercial or pellet filament; however, each of the three filament types on average saved the user over $50 \%$ of the cost of commercially available alternative toys. When items that cost more to print than purchase were removed, the average cost and percent savings were nearly $75 \%$ when using commercial filament and over $90 \%$ for pellet-extruded filament and recyclebot filament.

Table 3. Cost savings for individual toys (US\$) and percent (\%) savings.

\begin{tabular}{|c|c|c|c|c|c|c|}
\hline \multirow{2}{*}{ Design } & \multicolumn{3}{|c|}{ Cost Savings (USD) } & \multicolumn{3}{|c|}{ Percent Savings (\%) } \\
\hline & $\begin{array}{c}\text { Commercial } \\
\text { Filament }\end{array}$ & Pellets & Recyclebot & $\begin{array}{c}\text { Commercial } \\
\text { Filament }\end{array}$ & Pellets & Recyclebot \\
\hline Pokemon Go aimer & $\$ 4.33$ & $\$ 4.81$ & $\$ 4.90$ & $87 \%$ & $96 \%$ & $98 \%$ \\
\hline Clash of Clans barbarian & $\$ 18.49$ & $\$ 18.86$ & $\$ 18.93$ & $97 \%$ & $99 \%$ & $100 \%$ \\
\hline Voltron figure & $\$(20.27)$ & $\$ 1.55$ & $\$ 5.72$ & $-203 \%$ & $16 \%$ & $57 \%$ \\
\hline Overwatch tracer gun & $\$ 21.98$ & $\$ 25.30$ & $\$ 25.94$ & $83 \%$ & $95 \%$ & $98 \%$ \\
\hline Overwatch reaper mask & $\$ 16.92$ & $\$ 22.75$ & $\$ 23.86$ & $68 \%$ & $91 \%$ & $95 \%$ \\
\hline Star Wars AT-AT & $\$(9.07)$ & $\$ 2.88$ & $\$ 5.16$ & $-121 \%$ & $38 \%$ & $69 \%$ \\
\hline Last Word Destiny Hand Cannon & $\$ 91.09$ & $\$ 97.52$ & $\$ 98.74$ & $91 \%$ & $98 \%$ & $99 \%$ \\
\hline Overwatch D.VA Light Gun & $\$ 48.67$ & $\$ 53.23$ & $\$ 54.11$ & $88 \%$ & $97 \%$ & $98 \%$ \\
\hline Overwatch Reaper Shotgun & $\$ 33.85$ & $\$ 38.56$ & $\$ 39.46$ & $84 \%$ & $95 \%$ & $98 \%$ \\
\hline Batman cowl & $\$ 3.19$ & $\$ 5.77$ & $\$ 6.26$ & $47 \%$ & $85 \%$ & $93 \%$ \\
\hline Destiny Hawkmoon gun & $\$ 88.51$ & $\$ 96.79$ & $\$ 98.37$ & $89 \%$ & $97 \%$ & $98 \%$ \\
\hline Destiny thorn gun & $\$ 7.68$ & $\$ 15.11$ & $\$ 16.53$ & $43 \%$ & $84 \%$ & $92 \%$ \\
\hline Star Wars VII Storm Trooper Helmet & $\$(2.81)$ & $\$ 13.78$ & $\$ 16.95$ & $-14 \%$ & $68 \%$ & $84 \%$ \\
\hline Kylo Ren helmet & $\$(9.27)$ & $\$ 0.36$ & $\$ 2.20$ & $-227 \%$ & $9 \%$ & $54 \%$ \\
\hline Wall outlet shelf & $\$ 8.33$ & $\$ 9.53$ & $\$ 9.76$ & $83 \%$ & $95 \%$ & $98 \%$ \\
\hline Kylo Ren lightsaber & $\$ 12.26$ & $\$ 17.84$ & $\$ 18.91$ & $61 \%$ & $89 \%$ & $95 \%$ \\
\hline Blade Runner blaster & $\$ 6.95$ & $\$ 11.31$ & $\$ 12.14$ & $53 \%$ & $87 \%$ & $93 \%$ \\
\hline Fallout 3 T45-d helmet & $\$ 147.42$ & $\$ 175.18$ & $\$ 180.47$ & $79 \%$ & $94 \%$ & $97 \%$ \\
\hline Venus de Milo figurine & $\$ 8.32$ & $\$ 8.81$ & $\$ 8.90$ & $92 \%$ & $98 \%$ & $99 \%$ \\
\hline Warcraft Frostmourne sword & $\$ 103.08$ & $\$ 124.66$ & $\$ 128.77$ & $78 \%$ & $94 \%$ & $97 \%$ \\
\hline 3DR Iris+ quadcopter & $\$ 7.70$ & $\$ 12.16$ & $\$ 13.02$ & $55 \%$ & $88 \%$ & $94 \%$ \\
\hline Pieta figurine & $\$ 12.90$ & $\$ 15.77$ & $\$ 16.32$ & $76 \%$ & $93 \%$ & $97 \%$ \\
\hline P08 Luger gun & $\$ 75.65$ & $\$ 78.07$ & $\$ 78.53$ & $96 \%$ & $99 \%$ & $99 \%$ \\
\hline Game of Thrones iron throne & $\$ 12.24$ & $\$ 14.41$ & $\$ 14.83$ & $80 \%$ & $94 \%$ & $97 \%$ \\
\hline The Thinker figurine & $\$ 20.09$ & $\$ 21.70$ & $\$ 22.00$ & $90 \%$ & $97 \%$ & $99 \%$ \\
\hline Overwatch McCree Peacemaker gun & $\$ 62.69$ & $\$ 67.95$ & $\$ 68.96$ & $90 \%$ & $97 \%$ & $99 \%$ \\
\hline Fallout 4 Pipboy 3000 MkIV & $\$ 53.54$ & $\$ 69.01$ & $\$ 71.96$ & $71 \%$ & $92 \%$ & $96 \%$ \\
\hline Articulated lamp & $\$ 3.60$ & $\$ 6.75$ & $\$ 7.35$ & $45 \%$ & $85 \%$ & $92 \%$ \\
\hline Overwatch throwing star & $\$ 6.67$ & $\$ 6.90$ & $\$ 6.95$ & $95 \%$ & $99 \%$ & $99 \%$ \\
\hline Strong bolt & $\$ 18.92$ & $\$ 19.08$ & $\$ 19.11$ & $99 \%$ & $100 \%$ & $100 \%$ \\
\hline Mazigner Z Super Robot & $\$ 7.15$ & $\$ 9.20$ & $\$ 9.59$ & $72 \%$ & $92 \%$ & $96 \%$ \\
\hline Sombra pistol & $\$ 72.99$ & $\$ 81.65$ & $\$ 83.30$ & $86 \%$ & $96 \%$ & $98 \%$ \\
\hline Han Solo blaster & $\$ 4.88$ & $\$ 8.66$ & $\$ 9.38$ & $48 \%$ & $86 \%$ & $93 \%$ \\
\hline Overwatch D.VA headset & $\$ 17.41$ & $\$ 18.55$ & $\$ 18.77$ & $92 \%$ & $98 \%$ & $99 \%$ \\
\hline Overwatch Mercy staff & $\$ 122.99$ & $\$ 153.93$ & $\$ 159.83$ & $74 \%$ & $93 \%$ & $96 \%$ \\
\hline Overwatch Mercy blaster & $\$ 9.52$ & $\$ 16.71$ & $\$ 18.08$ & $49 \%$ & $86 \%$ & $93 \%$ \\
\hline Gears of War Chainsaw gun & $\$ 162.23$ & $\$ 189.46$ & $\$ 194.66$ & $81 \%$ & $95 \%$ & $97 \%$ \\
\hline Destiny Duke MK.44 gun & $\$ 117.11$ & $\$ 122.80$ & $\$ 123.89$ & $94 \%$ & $98 \%$ & $99 \%$ \\
\hline Fallout 4 Laser pistol & $\$ 57.91$ & $\$ 81.04$ & $\$ 85.46$ & $64 \%$ & $90 \%$ & $95 \%$ \\
\hline Overwatch loot box & $\$ 5.97$ & $\$ 8.14$ & $\$ 8.56$ & $66 \%$ & $91 \%$ & $95 \%$ \\
\hline Cat at British Museum & $\$ 74.49$ & $\$ 75.57$ & $\$ 75.78$ & $98 \%$ & $99 \%$ & $100 \%$ \\
\hline Halo 5 assault rifle & $\$(5.69)$ & $\$ 34.47$ & $\$ 42.14$ & $-11 \%$ & $69 \%$ & $84 \%$ \\
\hline Portal gun & $\$ 242.91$ & $\$ 269.65$ & $\$ 274.75$ & $87 \%$ & $96 \%$ & $98 \%$ \\
\hline Clash of Clans figurine & $\$ 29.94$ & $\$ 29.98$ & $\$ 29.98$ & $100 \%$ & $100 \%$ & $100 \%$ \\
\hline Harry Potter elder wand & $\$ 4.38$ & $\$ 4.67$ & $\$ 4.73$ & $91 \%$ & $98 \%$ & $99 \%$ \\
\hline Groot flower pot & $\$ 7.61$ & $\$ 8.60$ & $\$ 8.79$ & $85 \%$ & $96 \%$ & $98 \%$ \\
\hline Melted Darth Vader mask & $\$ 3.83$ & $\$ 6.84$ & $\$ 7.41$ & $48 \%$ & $85 \%$ & $93 \%$ \\
\hline Fallout $410 \mathrm{~mm}$ pistol & $\$ 5.75$ & $\$ 19.62$ & $\$ 22.27$ & $23 \%$ & $79 \%$ & $89 \%$ \\
\hline Starcraft Kerrigan statue & $\$ 53.07$ & $\$ 55.03$ & $\$ 55.41$ & $95 \%$ & $99 \%$ & $99 \%$ \\
\hline Destiny ghost & $\$ 4.19$ & $\$ 6.22$ & $\$ 6.60$ & $60 \%$ & $89 \%$ & $94 \%$ \\
\hline Lich king figurine & $\$ 3.18$ & $\$ 11.70$ & $\$ 13.33$ & $21 \%$ & $78 \%$ & $89 \%$ \\
\hline Ant Man helmet & $\$ 35.68$ & $\$ 42.11$ & $\$ 43.33$ & $80 \%$ & $94 \%$ & $97 \%$ \\
\hline Game of Thrones House emblem & $\$ 9.12$ & $\$ 9.72$ & $\$ 9.83$ & $92 \%$ & $98 \%$ & $99 \%$ \\
\hline
\end{tabular}


Table 3. Cont.

\begin{tabular}{|c|c|c|c|c|c|c|}
\hline \multirow{2}{*}{ Design } & \multicolumn{3}{|c|}{ Cost Savings (USD) } & \multicolumn{3}{|c|}{ Percent Savings (\%) } \\
\hline & $\begin{array}{l}\text { Commercial } \\
\text { Filament }\end{array}$ & Pellets & Recyclebot & $\begin{array}{l}\text { Commercial } \\
\text { Filament }\end{array}$ & Pellets & Recyclebot \\
\hline Clash of Clans king figure & $\$ 18.42$ & $\$ 18.84$ & $\$ 18.92$ & $97 \%$ & $99 \%$ & $100 \%$ \\
\hline Tooth toothbrush holder & $\$(0.91)$ & $\$ 1.19$ & $\$ 1.59$ & $-46 \%$ & $59 \%$ & $79 \%$ \\
\hline Halo 3 ODST helmet & $\$(17.14)$ & $\$ 2.42$ & $\$ 6.16$ & $-172 \%$ & $24 \%$ & $62 \%$ \\
\hline Fallout 4 protectron figure & $\$ 22.61$ & $\$ 24.33$ & $\$ 24.65$ & $90 \%$ & $97 \%$ & $99 \%$ \\
\hline Discobolus figurine & $\$ 6.40$ & $\$ 7.01$ & $\$ 7.13$ & $88 \%$ & $97 \%$ & $98 \%$ \\
\hline Anonymous mask & $\$(4.34)$ & $\$ 2.36$ & $\$ 3.64$ & $-88 \%$ & $48 \%$ & $73 \%$ \\
\hline Guardian of the Galaxy Star Lord mask & $\$(2.53)$ & $\$ 3.61$ & $\$ 4.79$ & $-42 \%$ & $60 \%$ & $80 \%$ \\
\hline Pokeball & $\$(4.36)$ & $\$(0.50)$ & $\$ 0.24$ & $-436 \%$ & $-50 \%$ & $24 \%$ \\
\hline Witcher 3 wall plaque & $\$ 42.57$ & $\$ 44.32$ & $\$ 44.66$ & $95 \%$ & $98 \%$ & $99 \%$ \\
\hline Overwatch widowmaker rifle & $\$ 112.79$ & $\$ 136.66$ & $\$ 141.22$ & $77 \%$ & $94 \%$ & $97 \%$ \\
\hline Fallout 4 combat rifle and shotgun & $\$ 76.48$ & $\$ 82.62$ & $\$ 83.80$ & $90 \%$ & $97 \%$ & $99 \%$ \\
\hline Michelangelo's David & $\$ 28.11$ & $\$ 29.42$ & $\$ 29.67$ & $94 \%$ & $98 \%$ & $99 \%$ \\
\hline Triceratops skull & $\$ 38.40$ & $\$ 39.55$ & $\$ 39.77$ & $96 \%$ & $99 \%$ & $99 \%$ \\
\hline Skull ring & $\$ 9.83$ & $\$ 9.92$ & $\$ 9.93$ & $99 \%$ & $100 \%$ & $100 \%$ \\
\hline Star Wars NN-44 Rey's Blaster & $\$ 41.58$ & $\$ 44.05$ & $\$ 44.52$ & $92 \%$ & $98 \%$ & $99 \%$ \\
\hline Joker mask & $\$(6.66)$ & $\$ 6.48$ & $\$ 8.99$ & $-58 \%$ & $56 \%$ & $78 \%$ \\
\hline Fork/spoon support for disability & $\$ 10.59$ & $\$ 10.88$ & $\$ 10.94$ & $96 \%$ & $99 \%$ & $99 \%$ \\
\hline Gryffindor coat of arms & $\$ 6.84$ & $\$ 7.68$ & $\$ 7.84$ & $85 \%$ & $96 \%$ & $98 \%$ \\
\hline Pokemon figurines & $\$ 28.47$ & $\$ 29.57$ & $\$ 29.78$ & $95 \%$ & $99 \%$ & $99 \%$ \\
\hline Rubik's cube & $\$ 4.70$ & $\$ 4.91$ & $\$ 4.95$ & $94 \%$ & $98 \%$ & $99 \%$ \\
\hline Fallout 4 Kellogg's pistol & $\$ 96.31$ & $\$ 98.97$ & $\$ 99.48$ & $96 \%$ & $99 \%$ & $99 \%$ \\
\hline Dobby the Elf figurine & $\$ 22.50$ & $\$ 28.89$ & $\$ 30.11$ & $72 \%$ & $92 \%$ & $96 \%$ \\
\hline Statue of Liberty figurine & $\$ 4.38$ & $\$ 5.86$ & $\$ 6.15$ & $68 \%$ & $91 \%$ & $95 \%$ \\
\hline Nutcracker & $\$ 3.51$ & $\$ 4.58$ & $\$ 4.78$ & $70 \%$ & $92 \%$ & $96 \%$ \\
\hline Minions figurines & $\$ 23.86$ & $\$ 25.98$ & $\$ 26.39$ & $89 \%$ & $97 \%$ & $98 \%$ \\
\hline Destiny sleeper simulant & $\$ 180.74$ & $\$ 203.04$ & $\$ 207.29$ & $85 \%$ & $96 \%$ & $98 \%$ \\
\hline Frozen Elsa figurine & $\$ 7.19$ & $\$ 7.77$ & $\$ 7.88$ & $90 \%$ & $97 \%$ & $99 \%$ \\
\hline Star Wars storm trooper rifle & $\$ 158.21$ & $\$ 166.71$ & $\$ 168.33$ & $93 \%$ & $98 \%$ & $99 \%$ \\
\hline Vitruvian Man sculpture & $\$ 31.20$ & $\$ 31.41$ & $\$ 31.45$ & $99 \%$ & $100 \%$ & $100 \%$ \\
\hline Robocop ED209 figure & $\$ 4.13$ & $\$ 17.01$ & $\$ 19.47$ & $19 \%$ & $77 \%$ & $89 \%$ \\
\hline Harry Potter wand & $\$ 13.36$ & $\$ 13.50$ & $\$ 13.52$ & $99 \%$ & $100 \%$ & $100 \%$ \\
\hline Cable guards & $\$ 1.08$ & $\$ 1.20$ & $\$ 1.23$ & $86 \%$ & $96 \%$ & $98 \%$ \\
\hline Game of Thrones dice cup & $\$ 6.89$ & $\$ 7.92$ & $\$ 8.12$ & $83 \%$ & $95 \%$ & $98 \%$ \\
\hline B2 bomber glider & $\$ 10.08$ & $\$ 10.43$ & $\$ 10.50$ & $95 \%$ & $99 \%$ & $99 \%$ \\
\hline Star Wars X Wing helmet & $\$ 18.37$ & $\$ 34.98$ & $\$ 38.15$ & $44 \%$ & $84 \%$ & $92 \%$ \\
\hline Hawkmoon \#2 & $\$ 84.92$ & $\$ 95.79$ & $\$ 97.86$ & $85 \%$ & $96 \%$ & $98 \%$ \\
\hline Destiny ghost \#2 & $\$ 5.84$ & $\$ 6.68$ & $\$ 6.84$ & $83 \%$ & $95 \%$ & $98 \%$ \\
\hline
\end{tabular}

Upon review, the items that cost more to print than to purchase (e.g., potentially lost the user money) when compared to commercially available and comparable products were often of noticeably higher value. For example, in Figure 1, the Game of Thrones inspired dice cup shown rendered in Cura is not commercially available but was compared to simple dice cups.

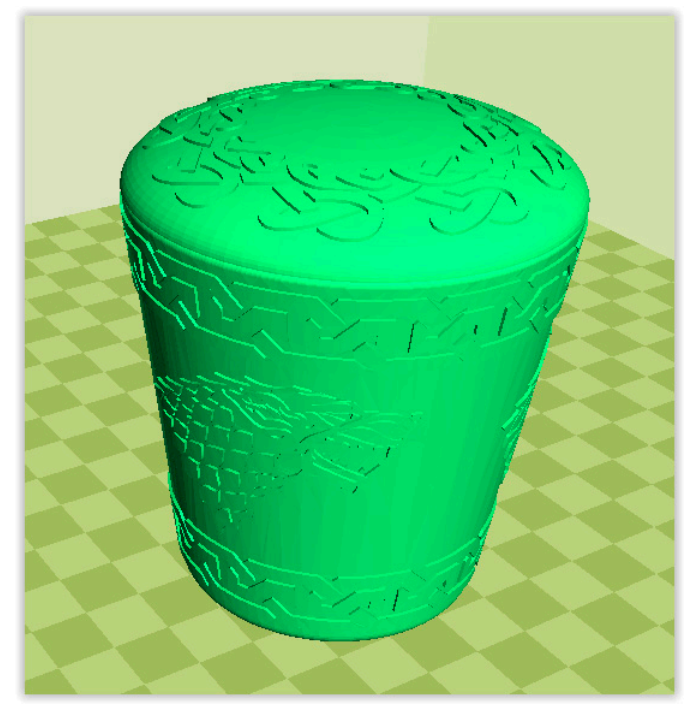

Figure 1. Cura rendering of details of freely available design of a Game of Thrones dice cup. 
The majority of the items that cost more to print than to purchase were specialized cosplay items. The term "cosplay" was added to the Oxford English Dictionary in 2008 as "the practice of dressing up as a character from a film, book, or video game, especially one from the Japanese genres of manga or anime" [48]. The appeal of cosplay is largely the expression of individualism within a shared community [49]. Not only does at-home additive manufacturing grant individual users the power to design and build completely customized items, but repositories such as MyMiniFactory provide an outlet for users to share designs and inspire one another in their creativity. Items in this study that did not save the user money when printed in all filaments such as the Voltron figure had dozens of specially designed parts that together made up a customized product of arguably significantly higher quality than the simple commercial alternative.

In addition, $6 \%$ of designs had no commercially available alternative either on Walmart.com nor offered by individual makers. Many of these were highly detailed cosplay items that came from specific games. While a cost saving could not be calculated, these items only highlight 3D printing's ability to allow users unlimited creativity in their work, and MyMiniFactory's data for these designs' downloads prove that these items are highly desired by the 3D printing and cosplaying community. Figure 2 shows the average percent savings of the cumulative designs with commercially available alternative elements based on the filaments used to print the items. Items printed with recyclebot-made filament demonstrated the greatest percent savings at $93 \%$ when all items were considered and $97 \%$ when only items that saved the user money were considered. It is clear that using recycled waste plastic would save consumers more than $90 \%$ of the costs of the conventionally-manufactured cost regardless of the circumstances. As $3 \mathrm{D}$ printing continues to become a more widely spread at-home activity and investment, filament technology may progress such that many users will utilize waste plastic as opposed to commercially available filament. Given the multi-billion status that is the toy industry, consumers may begin printing toys, games and specialty items in mass, saving substantial amounts of money in comparison to commercially available products.

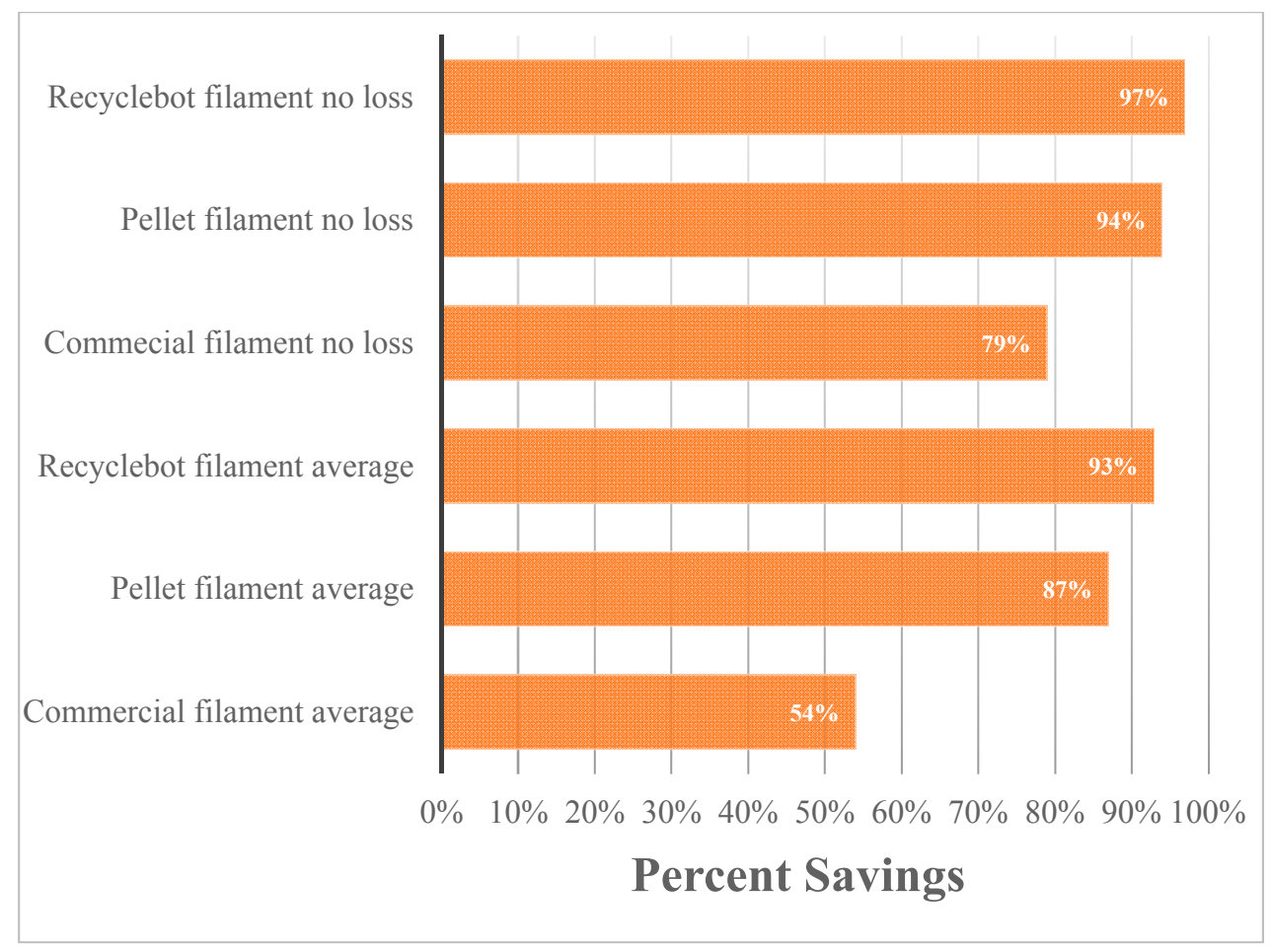

Figure 2. Bar chart showing the percent savings of printing designs with various filament types. 


\subsubsection{Macroeconomic Impact of Home Manufacturing}

The total results from Table 3 are the most striking. Over a single month period, MyMiniFactory saved its users more than $\$ 5$ million in avoided consumer purchases for only the top 100 downloaded designs. This assumes filament costs $\$ 20 / \mathrm{kg}$ or less, which is a reasonable assumption as the most popular filament is $\$ 23 / \mathrm{kg}$ and there are many commercial suppliers (even available on Amazon) that are selling filament for less than $\$ 20 / \mathrm{kg}$ as well as the much lower costs observed with pellet extruded filament or recyclebot manufactured filament. Extrapolated over a year, these results indicate that MyMiniFactory, just a single 3D printing repository among dozens, is saving consumers over $\$ 60$ million a year in offset purchases. Again, this value is conservative as many of the designs discarded from the analysis here would be more (not less) expensive to acquire commercially. In addition, this only considers 100 designs. MyMiniFactory currently has 26,355 published designs as of 30 April 2017 so this study only looked at $0.38 \%$. In addition, this represents, but a small fraction of the overall freely available designs, which is at least several million [21].

It can be safely concluded that the open source 3D printing community is already having more than $\$ 100$ million/year on the toy and game market. As the microeconomic savings are significant for individual consumers and the number of desktop 3D printer users continues to climb, this impact can be expected to grow.

\subsection{Six Example Printable Toys}

Although the toys can be made for less than purchased commercially, they may not be of the same value. Figure 3 shows the visual results of six common household toys to probe this effect. The free design 3D printable version is shown on the left and the commercial version in shown on the right. First, the mini travel chess set available from the largest online retailer, Amazon, results in a $90 \%$ savings if 3D printed. The color difference actually provides a visual advantage to 3D printing. Although it should be pointed out that identical (or different) color schemes could be enabled simply by using different colors of plastic filament for the different components of a toy. For those that find the wood more aesthetically appealing, there is already a wide range of wood filaments on the market and other biocomposites [50], and recent work indicates that even wood waste can be converted to 3D printer filament [51]. Home manufacturing of toys is also more economic when compared to a dedicated toy seller like ToysRUs. The shape puzzle available from ToysRUs results in a $88 \%$ savings and the toy truck $79 \%$ savings. In both cases, the commercial versions have a higher degree of color variance than shown, but this could be overcome by using more colors of filament and/or changing filaments during a single print. For more complex toys, like the action figures, 3D printing still results in a savings, although lower (e.g., $41 \%$ ) values. In addition to mass manufactured toys, wood toys made available on sites like Etsy can also be replaced with distributed manufacturing using 3D printing with substantial cost savings. For example, the wood puzzle star available on Etsy can be 3D printed for $82 \%$ savings as can the math spinner toy for $90 \%$ savings. It should be noted here that the relative cost savings can be heavily influenced not only by the filament selection but also the infill percentage. For example, when comparing 3D printed toys to wood based objects, the environmental impact can be lower when low infill settings are used, and, in general for non-solid plastic products, 3D printed ones have a lower impact than conventionally manufactured ones [52,53]. 


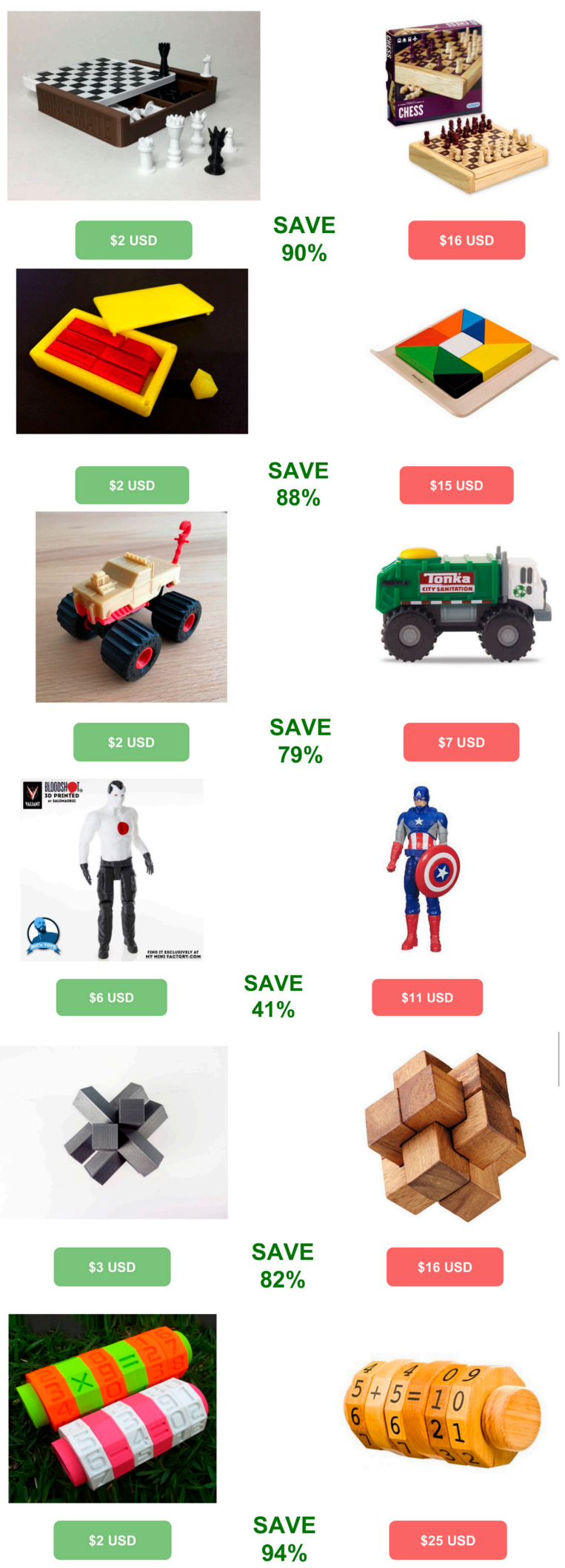

Figure 3. Visual comparison of open source 3D printed toys and their commercial mass manufactured equivalents. Costs and percent savings are shown for each toy.

Making a more careful comparison of some of the example toys shown in Figure 3, it is clear that these toys both appear and are actually different than the commercial counterparts (e.g., design, shape, 
and color) although the functionality (e.g., play) is similar or identical. The difference is perhaps most stark with the action figure (e.g., Captain America vs. BloodShot). There is currently no equivalent quality BloodShot action figure and the intellectual property surrounding the example is owned by Valliant Comics. The play with either action figure is identical (or nearly identical as super powers for both comic book characters arose from former soldiers being injected with super solider formula and nanites, respectively). However, a specific branded toy has a value to the consumer. This value can be acquired by the consumer by customizing the toy to fit their need (e.g., add minor changes to the existing action figure and/or repaint it) as well as print accessories such as a Captain America shield, which is already available on MyMiniFactory. The intellectual property concerns of home manufacturers doing such modifications are left for future work.

\subsection{Lego Analysis}

Lego is well known in the open source maker community (e.g., commons based peer production using a Lego-built 3D printing/Milling machine [54]). There are already hundreds of Lego designs available and customized OpenSCAD code generators for various Lego-compatible blocks. As can be seen in Figure 4, it is possible to fabricate Lego compatible bricks for less money than purchasing them with any type of ABS filament. It should be noted that the fit of the Lego blocks are superior to the generic compatible block and all of the 3D printed blocks on their first run. The fit of the 3D printed blocks, however, can be adjusted by the individual consumer to make blocks easier to disassemble (e.g., for weaker hands) or tighter (e.g., to make more permanent structures). The quality of the 3D printed parts can also be a key determinant in demand. Care must be taken by home toy manufacturers to ensure both the polymer used as well as the infill is appropriate for the toy being fabricated. In addition, there are some inherent limitations on the visual quality of FFF 3D printed parts. As can be seen in Figure 4, for the three unsmoothed 3D printed blocks lines can be observed on the $z$-axis. However, if this is important to the home user, ABS can be smoothed (bottom right block in Figure 4) with acetone, rendering a block very close to the visual quality of the generic block and removing print lines. In addition, 3D printed blocks can be made that are not available from Lego as shown as the example of the Lego to Lincoln Logs adapter blocks rendered by Cura [55] in Figure 5. It should be noted that several runs may be needed by the home manufacturer of Lego compatible blocks to obtain an ideal fit for the users, which would contributed to higher costs, although to a small degree. For example, it might take five tries to get the perfect fit, but then the settings could be used to print out a standard set of 1000 blocks so the trials needed would represent a minor loss and cost (e.g., $0.5 \%)$. For many other toys, such careful tolerances are not necessary.

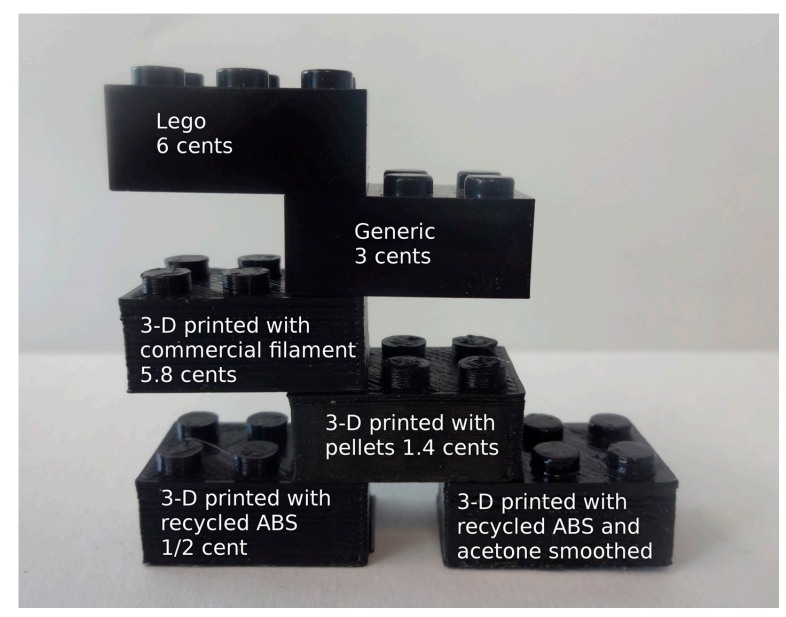

Figure 4. Photograph of $2 \times 3$ toy bricks: Lego brick, Lego-compatible generic brick, 3D printed commercial ABS and 3D printed recycled acrylonitrile butadiene styrene (ABS) bothnatural and acetone smoothed with costs. 


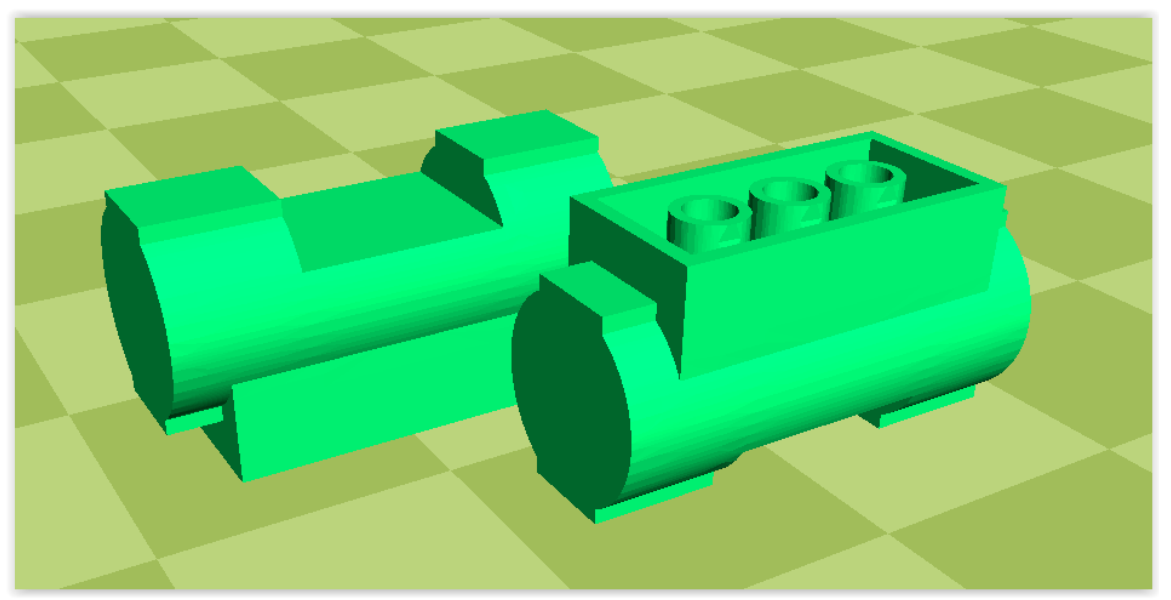

Figure 5. Adapterz LLC free design of a Lego to Lincoln Log adapter [55] shown in Cura.

$2 \times 3$ 3D printed Lego compatible blocks have a mass of $2.5 \mathrm{~g}$. To provide a fair comparison to a commercially available Lego-compatible generic brick set [56], the costs of fabricating $10002 \times 3$ Lego compatible bricks is shown with the various sources shown in Figure 6. In reviewing the top 10 most popular Lego sets on Walmart.com, it was found that the average cost per piece was $\$ 0.075$. The average cost for these kits on Amazon.com was $\$ 74.82$ with simple Lego blocks costing $\$ 60.00$. Generic building blocks cost less at $\$ 29.99$ [56]. When estimating the cost using 3D printed blocks, a comparable kit printed with commercial filament would cost $\$ 57.50$. A kit printed from pellets would cost $\$ 13.75$ and one printed from recyclebot-made filament would cost only $\$ 5.40$. In consideration of Wired's cost estimate of a single Lego brick [42], a 1000-piece kit would cost $\$ 104$, nearly double that of the kit printed with the more expensive commercial filament. Interestingly, with the cost of RepRap 3D printer kits now breaking the $\$ 100$ cost barrier (the Startt 3D printer from iMakr (London, England) is currently selling for \$99.99) [57], only roughly two sets of Legos need to be printed with pellet-made filament to recover the cost of the 3D printer. However, it should be noted that such kits still require substantial technical competence from the consumer.

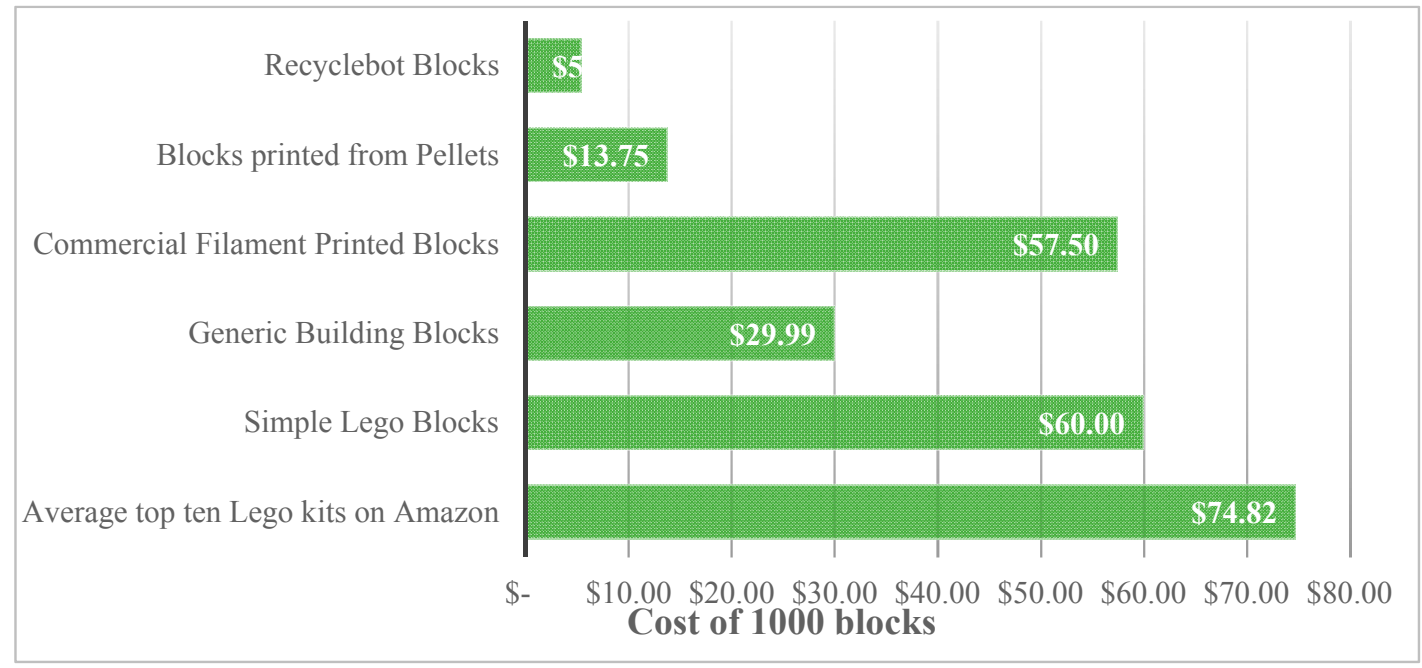

Figure 6. The costs of fabricating $10002 \times 3$ Lego compatible bricks or purchasing.

\subsection{Board Game}

Save the Planet Board Game is a free and open-source DIY cooperative board game [46] shown in Figure 7. In the game, players work together to save the planet to win the game while learning 
how to save the planet in real life. Users must 2D print out the game board and card decks for the beginner and advanced options, respectively. This can be done on $8 \times 11$ inch office paper ( $4 \times 11$ cents/page is 44 cents) and laminated to be glued to cereal boxes or printed directly on cardstock $(1 \times 69$ cents) so the total $2 \mathrm{D}$ printing cost is $\$ 1.13$. The beginner option has game pieces for simple acts of environmental benefit and is appropriate for children 4 and up and the advanced option is for teens and adults interested in more scalable impact. This game is open-source so like the RepRap, users are encouraged to build on it-make it better, add more "good deeds", make a local deed list and make more advanced derivatives.

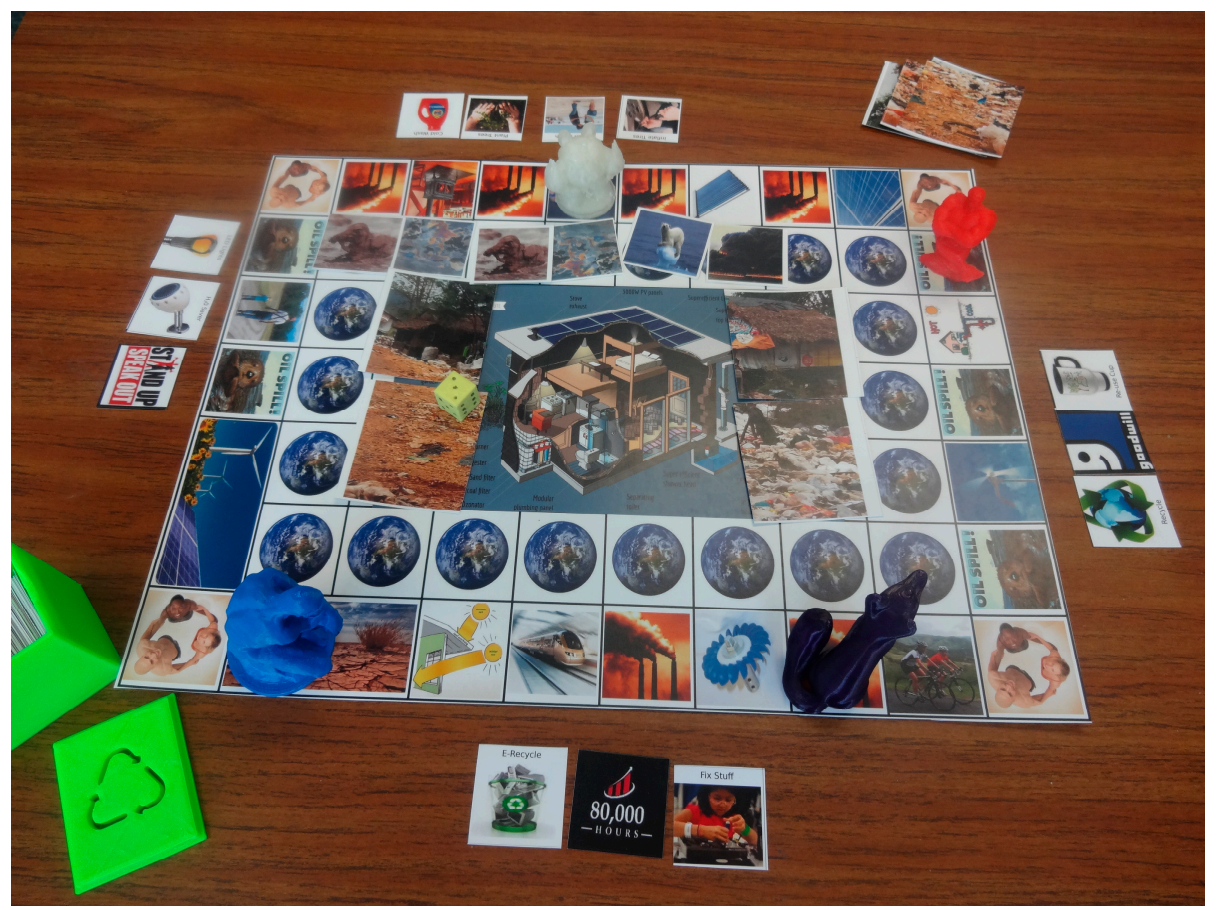

Figure 7. Open-source fully 2D and 3D printable do-it-yourself (DIY) cooperative Save the Planet Board Game in mid-play.

The following needs to be printed (summarized in Table 4): a card holder, dice and four mini-figures. The mini-figurines are completely up to the interests of the user and, with thousands of designs to choose from, enable some creativity of the user. There is also some flexibility in the size of the figures that will affect the cost and found items (e.g., a small stone) could reduce the cost of the figures to zero but is of lower value. Here, in order to demonstrate the range of options a fox, The Thinker at the Musée Rodin France, a figurine from another open board game, and a superman bust are used.

Table 4. 3D and 2D printed components, source and cost of Save the Planet Board Game assuming commercial filament and cardstock.

\begin{tabular}{cccc}
\hline Item & Source (myminifactory.com/object/) & Mass/Sheets & Cost [USD] \\
\hline Card holder & card-holder-for-save-the-planet-board-game-35620 & 39.15 & 0.90 \\
Dice & the-magnificent-dice-27043 & 1.68 & 0.04 \\
The Thinker & the-thinker-at-the-muse-rodin-france-2127 & 13.86 & 0.32 \\
Fox & fox-support-free-5865 & 8.04 & 0.18 \\
Open board game figurine & open-board-game-figurine-6013 & 7.50 & 0.17 \\
Superman bust & superman-bust-3518 & 6.46 & 0.15 \\
Game Boards & 2D printing costs & 5 sheets & 1.13 \\
\hline & & & 2.89 \\
\hline
\end{tabular}


The top 10 best-selling board games on Amazon as of 3 May 2017 averaged in price at $\$ 18.18$ with a range of $\$ 23.22$, where the most expensive game was $\$ 31.99$ and the least expensive was $\$ 8.77$ [47]. The 3D and 2D printed components, source and cost of Save the Planet Board Game costs less than $\$ 3$ as shown in Table 4, representing a $67 \%-91 \%$ savings. However, in this case, it is not an apple to apple comparison for identical games, which would come with a risk of intellectual property infringement. Instead, this analysis was for comparing a custom board game (with potentially greater value) to a generic board game. The closest analog of the example game is A Beautiful Place that costs $\$ 16.49$ on Amazon and is significantly less sophisticated, although similar in game play.

Already, many custom games are available in the open source community that have identical game play to conventionally manufactured and sold games, but are superior in some way. For example, many conventionally-manufactured BattleShip games are available on the market in the general price range of the most popular board games. All of them have relatively simple injection molded ships. A 3D printable game has been developed (battlefleet-star-wars-vs-star-trek-33840), which has identical game play but uses fan art mockups of Star Wars and Star Trek ships. The printed game also comes in 3D printable carrying cases for easy mobile play and is potentially of greater value than the Battleship games on the market for Star Wars/Star Trek fans.

\subsection{Discussion}

As the use of 3D printing has shifted from rapid prototyping in industry to production [58], research has shown DIY in-home manufacturing could easily justify the capital costs of a 3D printer with consumer items [21], and this study shows that even when a home printer is focused on making only games and toys, it is clear consumers can generate higher value items for less money than is currently available commercially. It should be pointed out here that these conclusions are in general conservative as this study focused only on relatively simple toys and games that required minimal assembly. Coupling open-source electronics to 3D printed toys (e.g., Arduino-driven 3D printable quad copters and VR headsets) enables far more sophisticated toys with higher values to be produced in the home. One area where this study was not conservative was in the estimation of failed print waste. Here, it was assumed to be zero as in the vast majority of cases printing a known 3D printable part (e.g., guaranteed 3D printable by MyMiniFactory) on an auto-calibrating/bed-leveling 3D printer (e.g., Lulzbot Taz or Mini) nearly always results in a successful print. This is not always the case, as a previous study using a home-built RepRap 3D printer estimated a $20 \%$ failure rate [20]. It should be noted, however, that this is only the case on the first prints from an inexperienced user on a much less sophisticated RepRap than are currently available to build, purchase assembled or buy in kit form. Future work could provide a more robust estimate of print failure rates by studying a large group of home 3D printer users.

3D printing gives consumers the unique ability to relatively easily fabricate products only for themselves, which may disrupt manufacturing in a wide array of markets [59-61]. Rifkin argues distributed manufacturing with $3 \mathrm{D}$ printers can lead to a zero marginal cost society [62]. It is unclear if the inconvenience of 3D printing yourself will not overcome the convenience of producing exceptionally low cost but high value bespoke products in one's own home; however, it is clear that it will have an impact on global value chains $[63,64]$. There have been a number of studies concluding that 3D printing will continue to have an increased impact on society $[65,66]$ both in the developed and developing world $[67,68]$. It is clear that 3D printing will play a major role in the rapidly emerging business models based on open source hardware [69] and open innovation [70]. In addition, even the humble toys here can have added value in the context of medicine when used as therapeutic aides (e.g., magnetic resonance imaging (MRI) visual demonstration tool [71]). Future open-source toy designs can also begin to take advantage of printing [72] or home milling electronic components [73], multimaterials [73,74] and 4D printing smart materials [75] to increase the toy complexity and playability. As the complexity increases, methods will be needed to ensure that such home manufactured toys meet known standards. Work in this area has already commenced [76]. In 
addition, there has been significant efforts targeting quality control for low-cost FFF 3D printers both informally [77-79] and formally [80-82] to enable real-time control, which when widespread will only enhance the conclusions in this study.

Future work is needed in both the technical areas to continue to reduce costs and improve reliability of open source 3D printers as well as to expand the economic analysis presented in this study. Future work can probe the time needed to design toys using open source software to determine ROIs for designers. In addition, more granular values can be obtained by doing sensitivity analysis on 3D printer user time for setting up a print and on routine maintenance (as well as associated costs). Future work is also needed to quantify the value to the prosumer of making his or her own toys. In this study, the value of the assembly time was considered to be zero as most of the toys were relatively simple to assemble. However, for more complex toys, there would be a time investment. Normal manufacturing considers this assembly time a cost, while, in this case, the assembly may be treated as part of the value of the toy itself in the same way that assembling a Lego kit is part of the game play. Finally, further work is needed to address the quality of recycling plastic for filament. This study showed that the largest savings could be found for distributed home manufacturing for products using recycled waste plastic filament. However, each time a thermoplastic is recycled, the mechanical properties are degraded. Cruz et al. have begun to investigate this phenomena's impact on open source 3D printing [83], but considerably more work is needed in the area over the complete array of polymers used in FFF.

With both the continued decrease in the cost of open source 3D printers and 3D printing filament along with the increase in the number and quality of free designs, it appears clear that consumer DIY manufacturing in the home [84] is set to have a significant impact on the toy and game markets in the future.

\section{Conclusions}

This study quantified the savings for consumers that utilize free and open source designs with a desktop fused filament 3D printer to fabricate their own toys and games. The cost of the filament was the largest variable controlling savings per product; however, each of the three filament types analyzed here on average saved the user over $75 \%$ of the cost of commercially available true alternative toys and over $90 \%$ for pellet-extruded filament and recyclebot-made filament. The reduced visual quality (e.g., 3D printer lines) was offset when compared to commercially available and comparable products because the 3D printable version could contain customization and increased complexity that created noticeably higher value for consumers. Over a single month period, MyMiniFactory saved its users more than $\$ 5$ million in avoided consumer purchases for only the top 100 downloaded designs. These results indicate that MyMiniFactory, just a single 3D printing repository among dozens, is saving consumers well over $\$ 60$ million a year in offset purchases. The specific case studies found that most common toy savings fell: $40 \%-90 \%$ in cost savings when using the most expensive filament. These cost savings came with ability to make never before seen toys. For example, although the cost of Lego blocks could be cut from 6 cents/block to about 0.5 cents per block using recycled filament, the real strength of $3 \mathrm{D}$ printing blocks is to make exactly what the consumer wants Lego compatible. Professional looking games fostering more creativity, customization and in-depth thought of the consumer can also be manufactured at home for a small fraction of purchasing them directly. The results of this study make it clear that consumers can generate higher value items for less money using an open source distributed manufacturing paradigm. With both the continued decrease in the cost of open source 3D printers and 3D printing filament along with the increase in the number and quality of free and open source designs, it appears clear that consumer DIY manufacturing is set to have a significant impact on the toy and game markets in the future.

Acknowledgments: The authors would like to thank MyMiniFactory for supplying data. 
Author Contributions: Joshua M. Pearce conceived and designed the study; Romain W. Kidd and Emily E. Petersen performed the data collection; Romain W. Kidd contributed data and Joshua M. Pearce contributed analysis tools; and all authors analyzed the data and wrote the paper. All authors have read and approved the final manuscript.

Conflicts of Interest: The authors declare no conflict of interest.

\section{Acronyms and Symbols}

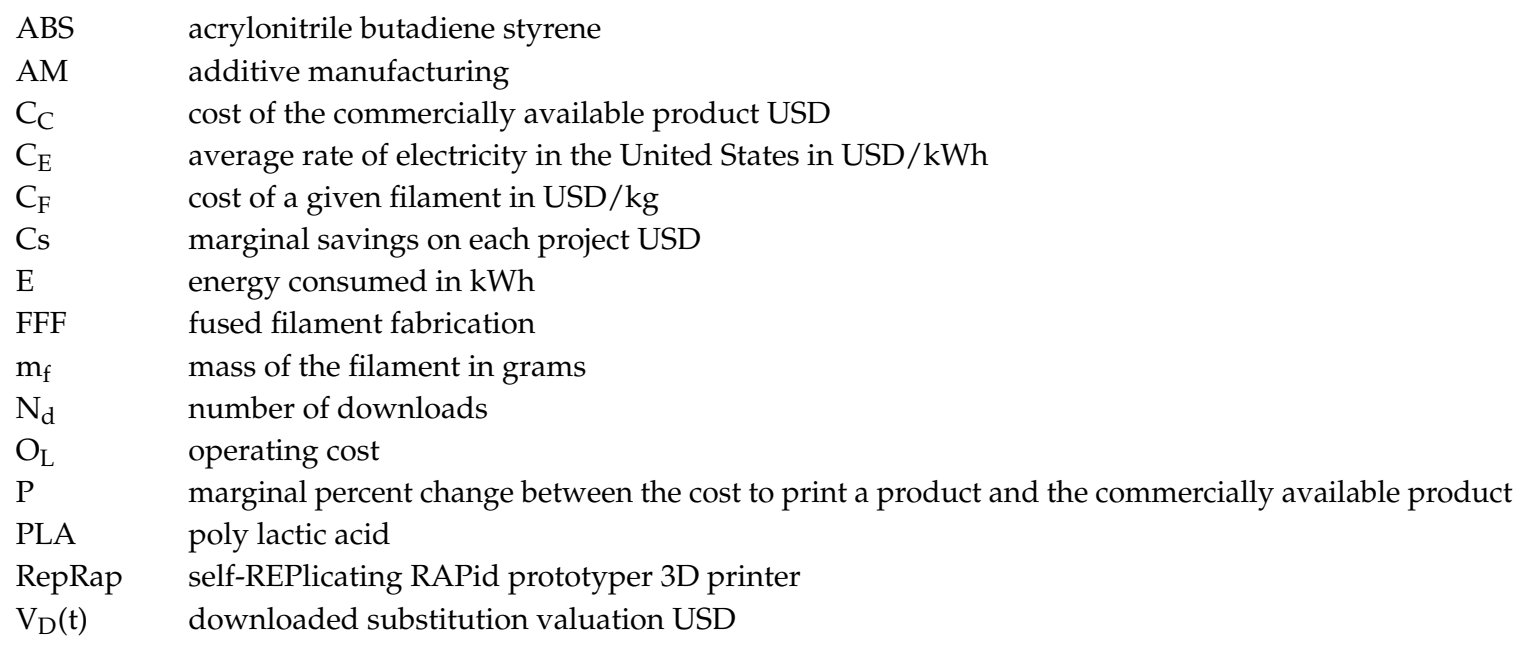

\section{References}

1. Sells, E.; Bailard, S.; Smith, Z.; Bowyer, A.; Olliver, V. RepRap: The Replicating Rapid Prototyper-Maximizing Customizability by Breeding the Means of Production. In Proceedings of the World Conference on Mass Customization and Personalization, Cambridge, MA, USA, 7-10 October 2007.

2. Jones, R.; Haufe, P.; Sells, E.; Iravani, P.; Olliver, V.; Palmer, C.; Bowyer, A. RepRap-the Replicating Rapid Prototyper. Robotica 2011, 29, 177-191. [CrossRef]

3. Bowyer, A. 3D Printing and Humanity's First Imperfect Replicator. 3D Print. Addit. Manuf. 2014, 1, 4-5. [CrossRef]

4. Gibb, A.; Abadie, S. Building Open Source Hardware: DIY Manufacturing for Hackers and Makers, 1st ed.; Addison-Wesley Professional: Boston, MA, USA, 2014.

5. Rundle, G. A Revolution in the Making; Simon and Schuster: New York, NY, USA, 2014.

6. Wohlers, T. Wohlers Report 2016: 3D Printing and Additive Manufacturing State of the Industry Annual Worldwide Progress Report; Wohlers Associates Inc.: Fort Collins, CO, USA, 2016.

7. Frauenfelder, M. Make: Ultimate Guide to 3D Printing 2014: Maker Media; O’Reilly Inc.: Sebastopol, CA, USA, 2013.

8. Moilanen, J.; Vaden, T. 3D Printing Community and Emerging Practices of Peer Production. First Monday 2013. [CrossRef]

9. Pearce, J.M. Building Research Equipment with Free, Open-Source Hardware. Science 2012, 337, $1303-1304$. [CrossRef] [PubMed]

10. Pearce, J. Open-Source Lab: How to Build Your Own Hardware and Reduce Research Costs, 1st ed.; Elsevier: Waltham, MA, USA, 2014.

11. Baden, T.; Chagas, A.; Marzullo, T.; Prieto-Godino, L.; Euler, T. Open Laware: 3-D Printing Your Own Lab Equipment. PLoS Biol. 2015, 13, e1002086.

12. Coakley, M.; Hurt, D.E. 3D Printing in the Laboratory Maximize Time and Funds with Customized and Open-Source Labware. J. Lab. Autom. 2016, 21, 489-495. [CrossRef] [PubMed]

13. Kentzer, J.; Koch, B.; Thiim, M.; Jones, R.W.; Villumsen, E.; May. An Open Source Hardware-Based Mechatronics Project: The Replicating Rapid 3-D Printer. In Proceedings of the 2011 4th International Conference on Mechatronics, Kuala Lumpur, Malaysia, 17-19 May 2011; pp. 1-8. 
14. Irwin, J.L.; Oppliger, D.E.; Pearce, J.M.; Anzalone, G. Evaluation of RepRap 3D Printer Workshops in K-12 STEM. In Proceedings of the 122nd ASEE Annual Conference and Exposition, Seattle, MA, USA, 14-17 June 2015.

15. Gonzalez-Gomez, J.; Valero-Gomez, A.; Prieto-Moreno, A.; Abderrahim, M. A new open source 3d-printable mobile robotic platform for education. In Advances in Autonomous Mini Robots; Springer: Berlin/Heidelberg, Germany, 2012; pp. 49-62.

16. Grujović, N.; Radović, M.; Kanjevac, V.; Borota, J.; Grujović, G.; Divac, D. 3D Printing Technology in Education Environment. In Proceedings of the 34th International Conference on Production Engineering, Nis, Serbia, 28-30 September 2011; pp. 29-30.

17. Schelly, C.; Anzalone, G.; Wijnen, B.; Pearce, J.M. Open-source 3-D printing technologies for education: Bringing additive manufacturing to the classroom. J. Visual Lang. Comput. 2015, 28, 226-237. [CrossRef]

18. Pearce, J.M.; Blair, C.; Laciak, K.J.; Andrews, R.; Nosrat, A.; Zelenika-Zovko, I. 3-D Printing of Open Source Appropriate Technologies for Self-Directed Sustainable Development. J. Sustain. Dev. 2010, 3, 17-29. [CrossRef]

19. Birtchnell, T.; Hoyle, W. 3D Printing for Development in the Global South: The 3D4D Challenge; Springer: Berlin, Germany, 2014.

20. Wittbrodt, B.; Glover, A.; Laureto, J.; Anzalone, G.; Oppliger, D.; Irwin, J.; Pearce, J. Life-Cycle Economic Analysis of Distributed Manufacturing with Open-Source 3-D Printers. Mechatronics 2013, 23, 713-726. [CrossRef]

21. Petersen, E.; Pearce, J. Emergence of Home Manufacturing in the Developed World: Return on Investment for Open-Source 3-D Printers. Technologies 2017, 5, 7. [CrossRef]

22. Kelleher, K. Was 3D Printing Just a Passing Fad? Time 2015. Available online: time.com/3916323/3d-printerstocks (accessed on 10 June 2015).

23. Bilton, R. 3D Printing Is a Gimmick, Says Foxconn Prez (and He's Sorta Right). 2013. Available online: https:/ /venturebeat.com/2013/06/26/3d-printing-is-a-gimmick-says-foxconn-prez-and-hes-sortaright/ (accessed on 1 July 2017).

24. Statistica. Average Amount Spent per Child on Toys by Country in 2013 (in U.S. Dollars). Available online: http:/ /www.statista.com/statistics/194424/amount-spent-on-toys-per-child-by-country-since-2009/ (accessed on 1 July 2017).

25. Brandongaille. 23 Toy Industry Statistics and Trends. 2013. Available online: http://brandongaille.com/23toy-industry-statistics-and-trends / (accessed on 1 July 2017).

26. Toy Association. Annual U.S. Sales Data. Available online: http://www.toyassociation.org/tia/ industry_facts/salesdata/industryfacts/sales_data/sales_data.aspx?hkey=6381a73a-ce46--4caf-8bc1-72b99567df1e\#.WQqVLYjys2w (accessed on 1 July 2017).

27. Global Industry Analysts, Inc. Press Release: Toys and Games-A Global Strategic Business Report. 2015. Available online: http:/ / www.strategyr.com/pressMCP-2778.asp (accessed on 1 July 2017).

28. LEGO: We Will Continue To Dominate The Global Toy Market. 2014. Available online: http:// www.businessinsider.com/lego-we-will-continue-to-dominate-the-global-toy-market-2014--2 (accessed on 1 July 2017).

29. Ultimaker. Software-All platforms. Available online: https://ultimaker.com/en/products/cura-software/ list (accessed on 1 July 2017).

30. Chilson, L. The Difference between ABS and PLA for 3D Printing. ProtoParadigm 2013. Available online: http://www.protoparadigm.com/news-updates/the-difference-between-abs-and-pla-for-3dprinting/ (accessed on 1 July 2017).

31. Stephens, B.; Azimi, P.; El Orch, Z.; Ramos, T. Ultrafine Particle Emissions from Desktop 3D Printers. Atmos. Environ. 2013, 79, 334-339. [CrossRef]

32. Tokiwa, Y.; Calabia, B.; Ugwu, C.; Aiba, S. Biodegradability of Plastics. Int. J. Mol. Sci. 2009, 10, 3722-3742. [CrossRef] [PubMed]

33. Hoffman, T. LulzBot Mini 3D Printer. PCMAG. 2015. Available online: http://www.pcmag.com/article2/0, 2817,2476575,00.asp (accessed on 1 July 2017).

34. U.S. Energy Information Administration Independent Short-Term Energy Outlook (STEO). Available online: https:/ / www.eia.gov/forecasts/steo/pdf/steo_full.pdf (accessed on 1 July 2017). 
35. Amazon Hatchbox 3D PLA-1kg 3.00-BLK PLA 3D Printer filament Dimensional Accuracy $\pm 0.05 \mathrm{Mm}, 1 \mathrm{Kg}$ Spool, 3.00 Mm, Black. Available online: http:/ / www.amazon.com/HATCHBOX-3D-PLA-1KG3--00-BLKFilament-Dimensional/dp/B00MEZE7XU (accessed on 1 July 2017).

36. Alibaba. PLA plastic pellets. Available online: https://www.alibaba.com/showroom/pla-plastic-pellets. html (accessed on 16 May 2017).

37. Baechler, C.; DeVuono, M.; Pearce, J.M. Distributed Recycling of Waste Polymer into RepRap Feedstock. Rapid Prototyp. J. 2013, 19, 118-125. [CrossRef]

38. Zhong, S.L.; Pearce, J.M. Tightening the Loop on the Circular Economy: Coupled Distributed Recycling and Manufacturing with Recyclebot and RepRap 3-D Printing. To be published.

39. Pearce, J. Quantifying the Value of Open Source Hardware Development. Mod. Econ. 2015, 6, 1-11. [CrossRef]

40. Pearce, J.M. Return on investment for open source scientific hardware development. Sci. Public Policy 2016, 43, 192-195. [CrossRef]

41. Wang, Z. Mattell: Buy the Toys While They're on Sale. 2015. Available online: http://seekingalpha.com/ article/3160396-mattel-buy-the-toys-while-theyre-on-sale (accessed on 1 July 2017).

42. Allain, R. How Much Does One Lego Piece Cost? 2014. Available online: https://www.wired.com/2014/ 08/lego-cost/ (accessed on 1 July 2017).

43. Griepp, M. Hobby Games Market Nearly \$1.2 Billion. ICv2. 2016. Available online: https://icv2.com/ articles/news/view/35150/hobby-games-market-nearly-1--2-billion (accessed on 1 July 2017).

44. Wong, J.I. Old-fashioned board games, not tech, are attracting the most money on Kickstarter. 2016. Available online: https:/ / qz.com/688843/old-fashioned-board-games-not-tech-are-attracting-the-most-money-onkickstarter/ (accessed on 1 July 2017).

45. Business Wire. Top 3 Trends Impacting the Global Board Games Market Through 2021: Technavio. 2016. Available online: http:/ / www.businesswire.com/news/home/20161228005057/en/Top-3-TrendsImpacting-Global-Board-Games (accessed on 1 July 2017).

46. Appropedia. Save the Planet Board Game. Available online: http://www.appropedia.org/Save_the_planet_ board_game (accessed on 1 July 2017).

47. Amazon Best Sellers. Board Games. Available online: https://www.amazon.com/Best-Sellers-Toys-GamesBoard/zgbs/toys-and-games/166225011 (accessed on 3 May 2017).

48. English Oxford Living Dictionaries- Cosplay. Available online: https://en.oxforddictionaries.com/ definition/cosplay (accessed on 4 May 2017).

49. Seregina, A.; Weijo, H. Play at Any Cost: How Cosplayers Produce and Sustain Their Ludic Communal Consumption Experiences. J. Consum. Res. 2017, 44, 139-159. [CrossRef]

50. Le Duigou, A.; Castro, M.; Bevan, R.; Martin, N. 3D printing of wood fibre biocomposites: From mechanical to actuation functionality. Mater. Des. 2016, 96, 106-114. [CrossRef]

51. Rudnicki, M.; Pringle, A.M.; Pearce, J.M. Viability of Up-Cycling Wood Furniture Waste to 3-D Printing Filament. In Advancements in Fiber-Polymer Composites Symposium; 2017, in press.

52. Kreiger, M.; Pearce, J.M. Environmental impacts of distributed manufacturing from 3-D printing of polymer components and products. In MRS Proceedings; Cambridge University Press: Cambridge, UK, 2013; Volume 1492, pp. 85-90.

53. Kreiger, M.; Pearce, J.M. Environmental life cycle analysis of distributed three-dimensional printing and conventional manufacturing of polymer products. ACS Sustain. Chem. Eng. 2013, 1, 1511-1519. [CrossRef]

54. Kostakis, V.; Papachristou, M. Commons-based peer production and digital fabrication: The case of a RepRap-based, Lego-built 3D printing-milling machine. Telemat. Inf. 2014, 31, 434-443. [CrossRef]

55. Adapterz LLC. Lego to Lincoln Logs. Available online: https://www.myminifactory.com/object/lego-tolincoln-logs-uck-05f06m-14924 (accessed on 5 May 2017).

56. Amazon. Building Bricks 0 Regular Colors-1000 Pieces-Compatible with All Major Brands. Available online: https:/ / www.amazon.com/Building-Bricks-Regular-Colors-Compatible/dp/B015EQIOCA/ref= sr_1_1 (accessed on 1 July 2017).

57. STARTT 3D Printer. iMakr.com. Available online: https://www.imakr.com/us/en/startt-affordable-3dprinter/1146-startt-3d-printer.html (accessed on 1 July 2017).

58. Bak, D. Rapid prototyping or rapid production? 3D printing processes move industry towards the latter. Assem. Autom. 2003, 23, 340-345. [CrossRef] 
59. Petrick, I.J.; Simpson, T.W. 3D printing disrupts manufacturing: How economies of one create new rules of competition. Res.-Technol. Manag. 2013, 56, 12-16. [CrossRef]

60. Berman, B. 3-D printing: The new industrial revolution. Bus. Horiz. 2012, 55, 155-162. [CrossRef]

61. Kietzmann, J.; Pitt, L.; Berthon, P. Disruptions, decisions, and destinations: Enter the age of 3-D printing and additive manufacturing. Bus. Horiz. 2015, 58, 209-215. [CrossRef]

62. Rifkin, J. The Zero Marginal Cost Society: The Internet of Things, the Collaborative Commons, and the Eclipse of Capitalism; Palgrave Macmillan: Basingstoke, UK, 2014.

63. Laplume, A.O.; Petersen, B.; Pearce, J.M. Global value chains from a 3D printing perspective. J. Int. Bus. Stud. 2016, 47, 595-609. [CrossRef]

64. Rehnberg, M.; Pointe, S. 3D Printing and Global Value Chains: How a New Technology May Restructure Global Production; Global Production Networks Centre: Singapore, 2016; GPN2016-010; Available online: http:/ /gpn.nus.edu.sg/file/Stefano\%20Ponte_GPN2016_010.pdf (accessed on 1 July 2017).

65. Pîrjan, A.; Petrosanu, D.M. The Impact of 3D Printing Technology on the Society and Economy. J. Inf. Syst. Oper. Manag. 2013. Available online: ftp://ftp.repec.org/opt/ReDIF/RePEc/rau/jisomg/Wi13/JISOMWI13-A19.pdf (accessed on 7 July 2017).

66. Thiesse, F.; Wirth, M.; Kemper, H.G.; Moisa, M.; Morar, D.; Lasi, H.; Piller, F.; Buxmann, P.; Mortara, L.; Ford, S.; Minshall, T. Economic Implications of Additive Manufacturing and the Contribution of MIS. Bus. Inf. Syst. Eng. 2015, 57, 139. [CrossRef]

67. Aitken-Palmer, W. A Market-Based Approach to 3d Printing for Economic Development in Ghana. Master's Thesis, Michigan Technological University, Houghton, MI, USA, 2015.

68. Pearce, J.M. Applications of open source 3-D printing on small farms. Org. Farming 2015, 1, 19-35. [CrossRef]

69. Pearce, J.M. Emerging Business Models for Open Source Hardware. J. Open Hardw. 2017, 1, 2. [CrossRef]

70. Reed, R.; Storrud-Barnes, S.; Jessup, L. How open innovation affects the drivers of competitive advantage: Trading the benefits of IP creation and ownership for free invention. Manag. Decis. 2012, 50, 58-73. [CrossRef]

71. Peh, Z.K.; Yap, Y.L.; Yeong, W.Y.; Liow, H.H. Application of 3D printed medical aid for pediatric cancer patients. In Proceedings of the 2nd International Conference on Progress in Additive Manufacturing, Nanyang Technological University, Singapore, 16-19 May 2016; pp. 49-54.

72. Saengchairat, N.; Tran, T.; Chua, C.K. A review: Additive manufacturing for active electronic components. Virtual Phys. Prototyp. 2017, 12, 31-46. [CrossRef]

73. Anzalone, G.C.; Wijnen, B.; Pearce, J.M. Multi-material additive and subtractive prosumer digital fabrication with a free and open-source convertible delta RepRap 3-D printer. Rapid Prototyp. J. 2015, 21, 506-519. [CrossRef]

74. Vidimče, K.; Wang, S.P.; Ragan-Kelley, J.; Matusik, W. OpenFab: A programmable pipeline for multi-material fabrication. ACM Trans. Graph. 2013, 32, 136. [CrossRef]

75. Khoo, Z.X.; Teoh, J.E.M.; Liu, Y.; Chua, C.K.; Yang, S.; An, J.; Leong, K.F.; Yeong, W.Y. 3D printing of smart materials: A review on recent progresses in 4D printing. Virtual Phys. Prototyp. 2015, 10, 103-122. [CrossRef]

76. Cruz Sanchez, F.A.; Boudaoud, H.; Muller, L.; Camargo, M. Towards a standard experimental protocol for open source additive manufacturing: This paper proposes a benchmarking model for evaluating accuracy performance of 3D printers. Virtual Phys. Prototyp. 2014, 9, 151-167. [CrossRef]

77. Gewirtz, D. Adding a Raspberry Pi Case and a Camera to Your LulzBot Mini-Watch Video Online-Watch Latest Ultra HD 4K Videos Online. 2016. Available online: http:/ /www.zdnet.com/article/ 3d-printing-hands-on-adding-a-case-and-a-camera-to-the-raspberry-pi-and-lulzbot-mini/ (accessed on 30 November 2016).

78. Printer3D. Free IP Camera Monitoring for 3D Printer with Old Webcam usb in 5min-3D Printers English French \& FAQ Wanhao Duplicator D6 Monoprice Maker Ultimate \& D4, D5, Duplicator 7, 2017. Available online: http:/ / www.printer3d.one/en/forums / topic/free-ip-camera-monitoring-for-3d-printer-with-oldwebcam-usb-in-5min/ (accessed on 18 March 2017).

79. Simon, J. Monitoring Your 3D Prints I3D Universe. 2017. Available online: https://3duniverse.org/2014/ 01/06/monitoring-your-3d-prints / (accessed on 18 March 2017).

80. Nuchitprasitchai, S.; Roggemann, M.; Pearce, J. Factors Effecting Real Time Optical Monitoring of Fused Filament 3-D Printing. Prog. Addit. Manuf. 2017, 1-17. [CrossRef]

81. Nuchitprasitchai, S.; Roggemann, M.; Pearce, J. Three Hundred and Sixty Degree Real-Time Monitoring of 3-D Printing Using Computer Analysis of Two Camera Views. J. Manuf. Mater. Process. 2017, 1, 2. [CrossRef] 
82. Nuchitprasitchai, S.; Roggemann, M.; Pearce, J. An Open Source Algorithm for Reconstruction 3-D images for Low-cost, Reliable Real-time Monitoring of FFF-based 3-D Printing. To be published.

83. Cruz, F.; Lanza, S.; Boudaoud, H.; Hoppe, S.; Camargo, M. Polymer Recycling and Additive Manufacturing in an Open Source context: Optimization of Processes and Methods. In Proceedings of the 2015 Annual International Solid Freeform Fabrication Symposium-An Additive Manufacturing Conference, Austin, TX, USA, 7-9 August 2015; pp. 10-12.

84. Rayna, T.; Striukova, L. From rapid prototyping to home fabrication: How 3D printing is changing business model innovation. Technol. Forecast. Soc. Chang. 2016, 102, 214-224. [CrossRef]

(C) 2017 by the authors. Licensee MDPI, Basel, Switzerland. This article is an open access article distributed under the terms and conditions of the Creative Commons Attribution (CC BY) license (http:/ / creativecommons.org/licenses/by/4.0/). 